Cahiers « Mondes anciens »

ANCIENS

Histoire et anthropologie des mondes anciens

$11 \mid 2018$

La « civilisation » : critiques épistémologique et historique

\title{
Alexandre et Octavien contre Bismarck et Gengis Khan
}

Les usages (problématiques) de l'Antiquité gréco-romaine dans l'univers ludique de Civilization

Alexander and Octavian against Bismarck and Gengis Khan. The (problematic) use of Greco-Roman Antiquity in the ludic world of Civilization

\section{Emmanuelle Valette}

\section{OpenEdition}

Journals

Édition électronique

URL : http://journals.openedition.org/mondesanciens/2101

DOI : 10.4000/mondesanciens. 2101

ISSN : 2107-0199

Éditeur

UMR 8210 Anthropologie et Histoire des Mondes Antiques

Référence électronique

Emmanuelle Valette, «Alexandre et Octavien contre Bismarck et Gengis Khan », Cahiers « Mondes anciens » [En ligne], 11 | 2018, mis en ligne le 19 mars 2018, consulté le 05 mai 2019. URL : http:// journals.openedition.org/mondesanciens/2101; DOI : 10.4000/mondesanciens.2101

Ce document a été généré automatiquement le 5 mai 2019.

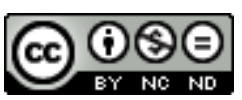

Les Cahiers «Mondes Anciens " sont mis à disposition selon les termes de la licence Creative Commons Attribution - Pas d'Utilisation Commerciale - Pas de Modification 4.0 International. 


\section{Alexandre et Octavien contre Bismarck et Gengis Khan}

Les usages (problématiques) de l'Antiquité gréco-romaine dans l'univers ludique de Civilization

Alexander and Octavian against Bismarck and Gengis Khan. The (problematic) use of Greco-Roman Antiquity in the ludic world of Civilization

Emmanuelle Valette 
Fig. 1a : Civilization $V$, un monde uchronique.

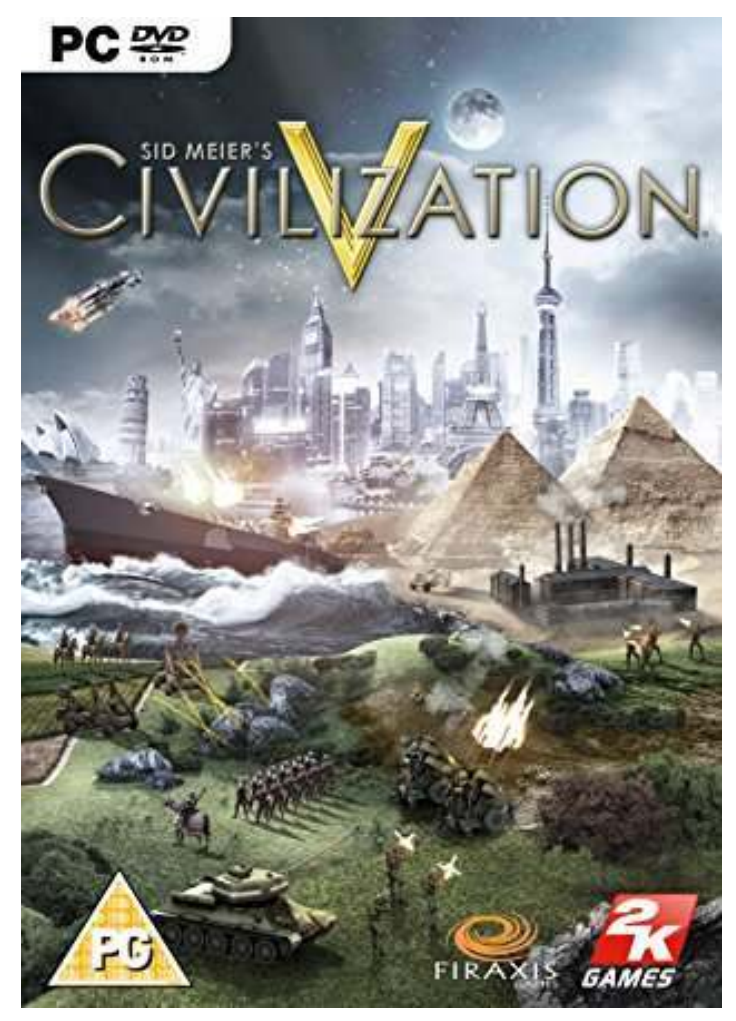

Jaquette avant du jeu Civilization V. (C) 2K Games | Firaxis

De nombreux jeux vidéo utilisent l'Antiquité dans leur scénario. Il y a là une forme de paradoxe $^{1}$ : plus la culture gréco-latine tend à disparaître des savoirs transmis à l'école, plus elle affiche sa vitalité dans diverses formes de "culture pop ${ }^{2}$ » (univers vidéoludique, mode, publicité, cinéma, séries TV). Or, tandis que certains jeux sont clairement centrés sur l'Antiquité gréco-romaine, son histoire, ses mythes, ses héros légendaires ${ }^{3}$, d'autres l'utilisent de façon plus implicite, comme univers de référence. Parmi ces derniers, un jeu passionne " gamers » et internautes depuis maintenant vingt-cinq ans : « Civilization $»^{4}$. Imaginé par Sid Meyer et développé par une société de production américaine, Firaxis Games, ce jeu est devenu, au fil des ans, une véritable série culte (fig. 1a). Il en est aujourd'hui à sa sixième version, sans compter la sortie de multiples « extensions » et compléments ${ }^{5}$. Ces évolutions permanentes répondent évidemment à une logique commerciale très concurrentielle : il faut maintenir l'intérêt des adeptes du jeu, capter de nouvelles cibles. Mais elles montrent aussi la nécessité de s'adapter au contexte culturel et social. Et pour qui s'intéresse à "l'idéologie du jeu », l'analyse des modifications dans le scénario et dans les règles du jeu est très révélatrice des interactions entre le « monde réel » et l'univers ludique : Civilization reflète les opinions et les préoccupations des producteurs et des consommateurs, de ceux qui créent le jeu et de ceux qui l'attendent, puis l'achètent. 
Fig. 1b : Octavien et Napoléon s'entretiennent de la tactique à adopter.

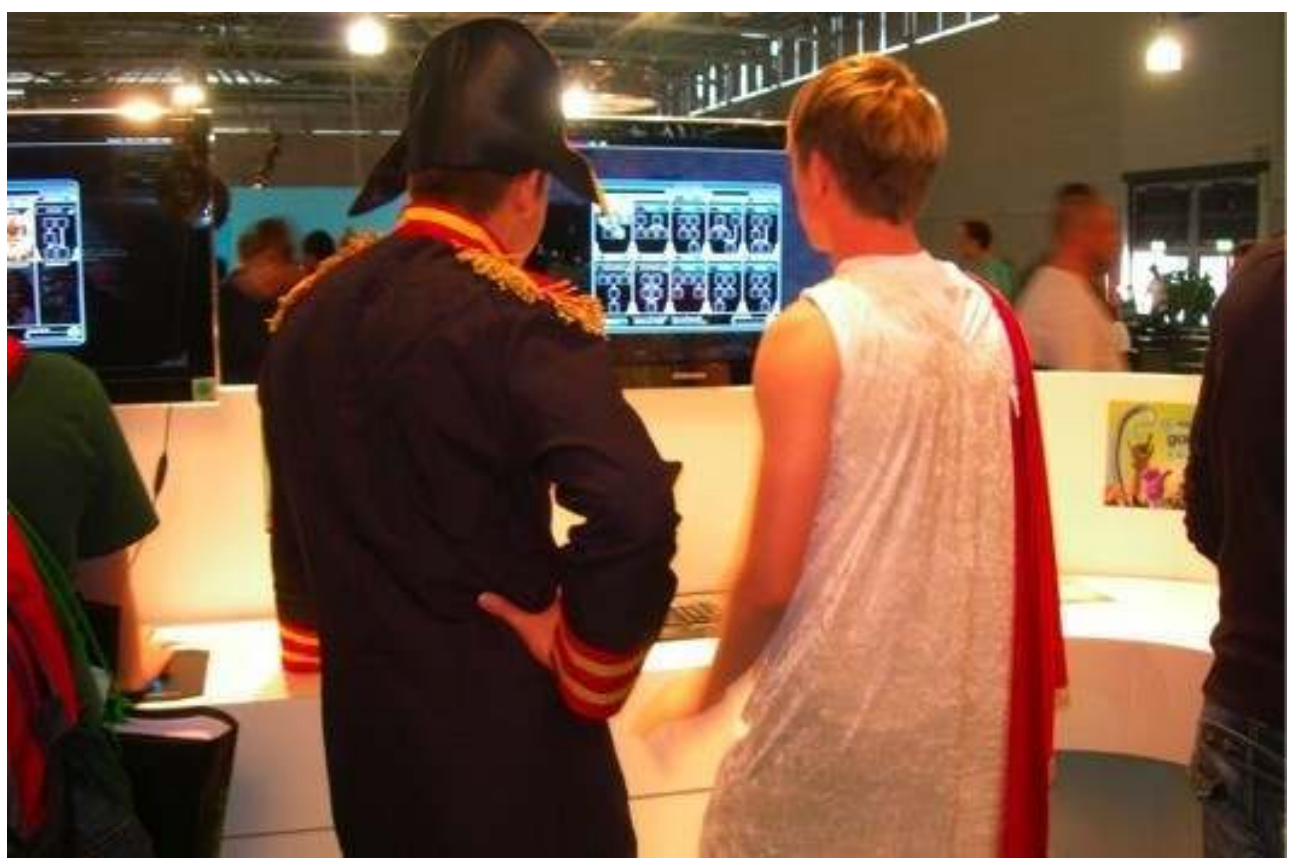

Capture d'écran (screenshot) Civilization V. () 2K Games | Firaxis

2 Le principe ? Conduire une "civilisation » depuis «l'âge de pierre » (stone age) jusqu'à l'époque contemporaine ${ }^{6}$, en choisissant parmi un large éventail de "civilisations" proposées et en affrontant d'autres civilisations ${ }^{7}$. Chaque étape de cette "évolution " permet d'acquérir de nouveaux " pouvoirs » et de " progresser » jusqu'à la victoire. Très sophistiqué du point de vue technologique ${ }^{8}$, Civilization se présente, aux yeux du néophyte ou de l'historien, comme une sorte de bric-à-brac culturel. Saturé de références historiques précises (par le nom des personnages, des lieux, des civilisations, des monuments), le jeu est en même temps dénué de toute vraisemblance historique, puisque le joueur peut se prendre pour ${ }^{9}$ Napoléon et affronter Octavien et Ramsès II (fig. 1b), la reine Victoria s'allier aux Hittites pour battre le Congo. L'univers ludique de Civilization fabrique en effet une forme d'uchronie ${ }^{10}$, constitutive du plaisir du jeu.

Par ailleurs, ce jeu s'appuie sur un évolutionnisme et un ethnocentrisme décomplexés, car tout en s'affranchissant de la "réalité historique», il installe un temps linéaire, orienté et structuré en fonction d'une chronologie calquée sur la périodisation de l'histoire traditionnelle. Chaque civilisation progresse en effet "d'ère en ère $»^{11}$, des temps les plus obscurs jusqu'à la modernité. D'autre part, cette marche continue vers le progrès est étroitement liée à l'acquisition de nouvelles techniques : Rome aura internet au terme de son évolution; les États-Unis devront, comme elle, inventer l'écriture et la navigation avant de pouvoir se lancer dans la conquête de l'espace. "L'invention technologique » joue donc un rôle central dans le scénario du jeu et dans la conception de la civilisation. 
Fig. 2 : : Alexandre le Grand, dirigeant de l'Empire grec dans Civilization V.

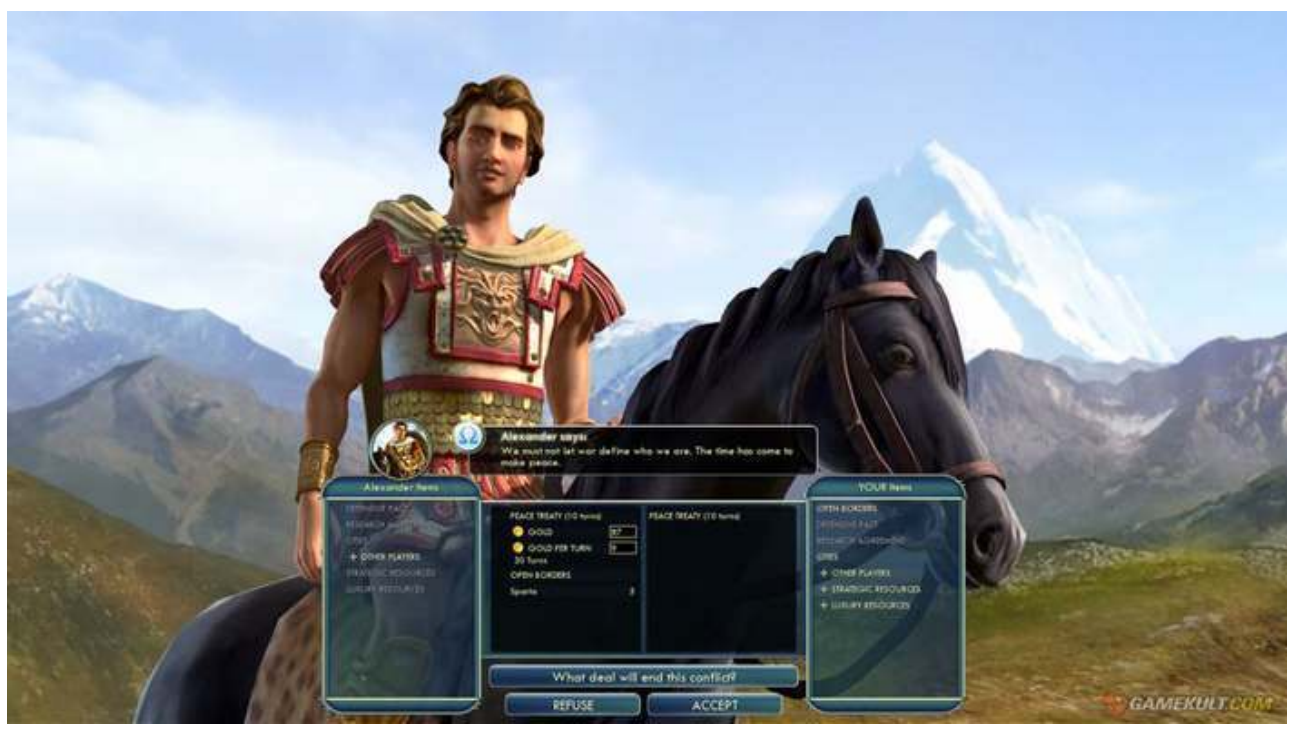

Capture d'écran (C2K Games | Firaxis

Enfin, ce jeu affiche un impérialisme triomphant et il porte à son comble l'idéologie du « choc des civilisations $»^{12}$. Parmi les dirigeants qui « conduisent » chaque civilisation vers la victoire, nombreuses en effet sont les figures de conquérants: Alexandre (fig. 2), Napoléon, Bismarck, Attila, etc. Et, certes, on pourra dire que dans Civilization, la guerre n'est qu'un moyen de gagner parmi d'autres, puisque la "victoire militaire " ne représente que l'une des cinq ou six options proposées au joueur en début de partie ${ }^{13}$, mais quel que soit le mode de jeu choisi, il s'agit toujours de bâtir un "empire » plus puissant que le voisin, pour le dépasser, le dominer et l'écraser ${ }^{14}$.

5 S'il en simplifie beaucoup la complexité, ce court résumé des principes du jeu et de son scénario permet de comprendre que Civilization participe ainsi à la création et à la transmission de stéréotypes, d'une part sur ce qui constitue « une civilisation» dans la pensée occidentale contemporaine, d'autre part sur la spécificité et sur l'apport supposé de chaque civilisation (ou de chaque peuple) à l'histoire universelle, et enfin sur la place conférée à l'Antiquité gréco-romaine dans le "grand récit » de l'Histoire de l'humanité. Ce sont ces questions qui nous intéresseront ici.

\section{Qu'est-ce qu'une « civilization »?}

6 La première question est celle de la définition : qu'entend le jeu par «civilisation »? Le choix de ce terme pour titre de la série montre que la notion est au centre du scénario. Si la longue notice (200 pages) qui accompagne chaque version du jeu ${ }^{15}$ ne fournit pas de véritable définition, la liste et surtout la description des civilisations proposées dans chaque nouvelle édition donnent un bon aperçu des critères retenus par les concepteurs du jeu. 


\section{Des listes hétérogènes}

7 Tout d'abord, la lecture attentive de ces listes montre l'hétérogénéité des éléments qui les composent. Le jeu mêle des civilisations anciennes (les Sumériens, l'Égypte) ou bien disparues (les Aztèques, les Mayas) et des civilisations « modernes » (comme la France ou les États-Unis). Cette disparité est surtout visible dans les noms choisis pour désigner ces civilisations : tantôt on fait référence à un peuple, une réalité ethnique (les Celtes, les Assyriens), tantôt à un espace géographique, une région (comme l'Arabie) ou une cité (comme Byzance ou Carthage). Une «tribu» (tribe), comme les Huns, voisine avec des États-Nations, comme la France, l'Espagne ou la Norvège ${ }^{16}$. Ce flou et cette hétérogénéité nous rappellent le caractère à la fois construit et très arbitraire de la notion de civilisation ${ }^{17}$. Le jeu ne fait que reprendre des catégories qui, pour la plupart, font partie de notre culture et de notre imaginaire partagé.

Fig. 3a : Shaka, le chef de la civilisation Zoulou (Civilization V)

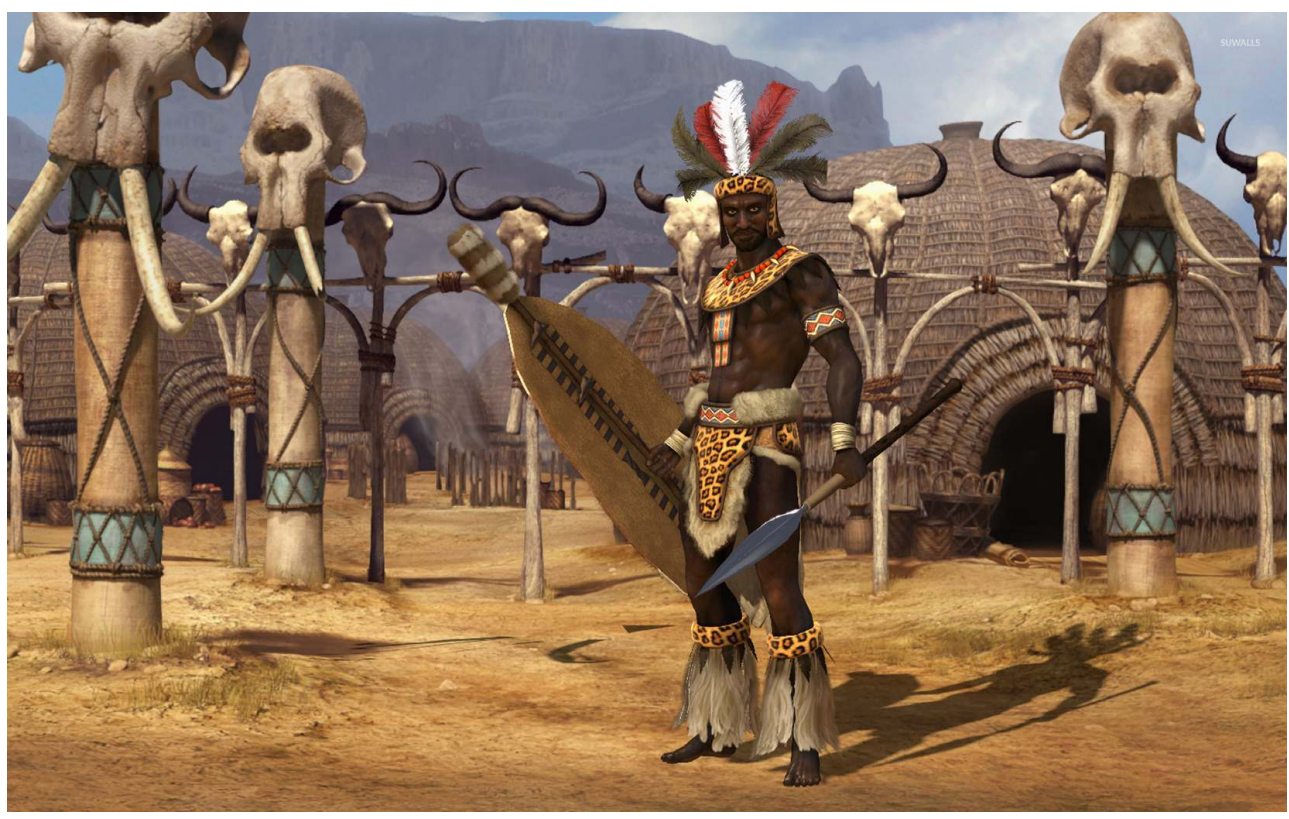

Capture d'écran @2K Games | Firaxis 
Fig. 3b : Hiawatha, dirigeant des Iroquois (Civilization V)

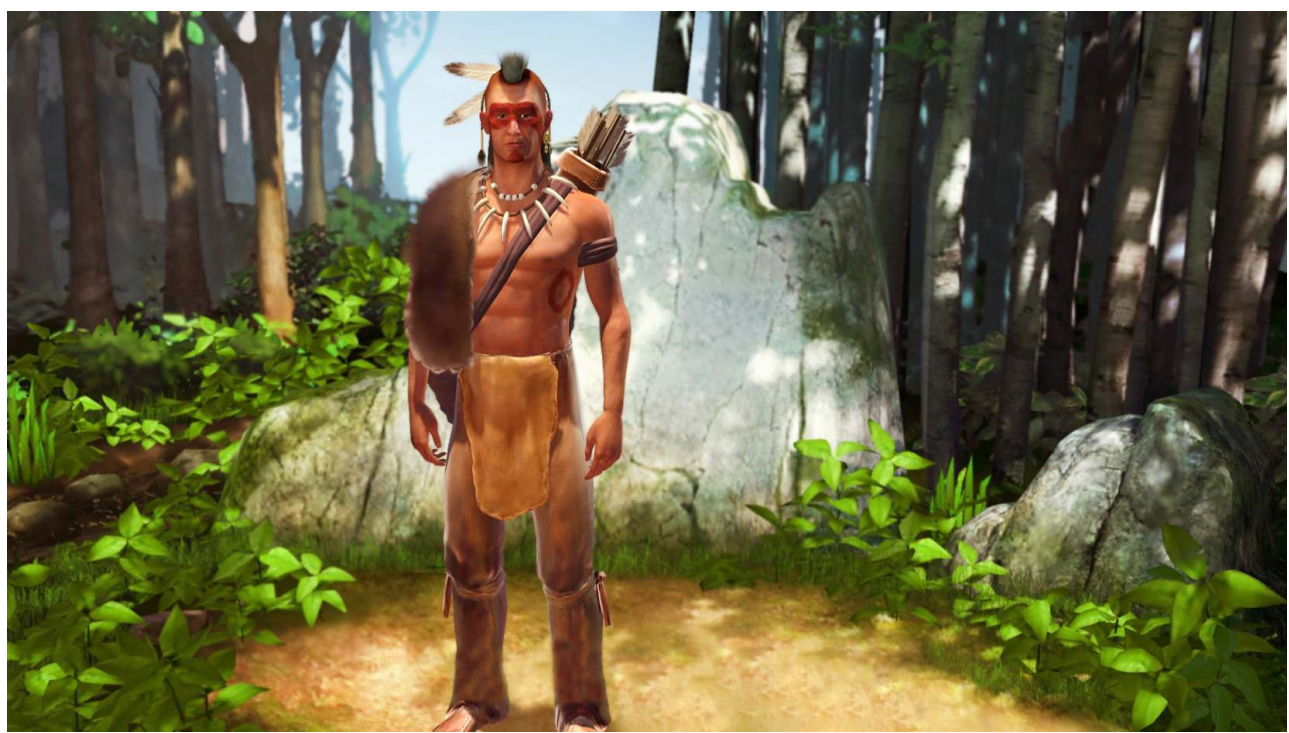

Capture d'écran @2K Games | Firaxis

Fig. 4a : Kamehameha 1er, le dirigeant de la Polynésie.

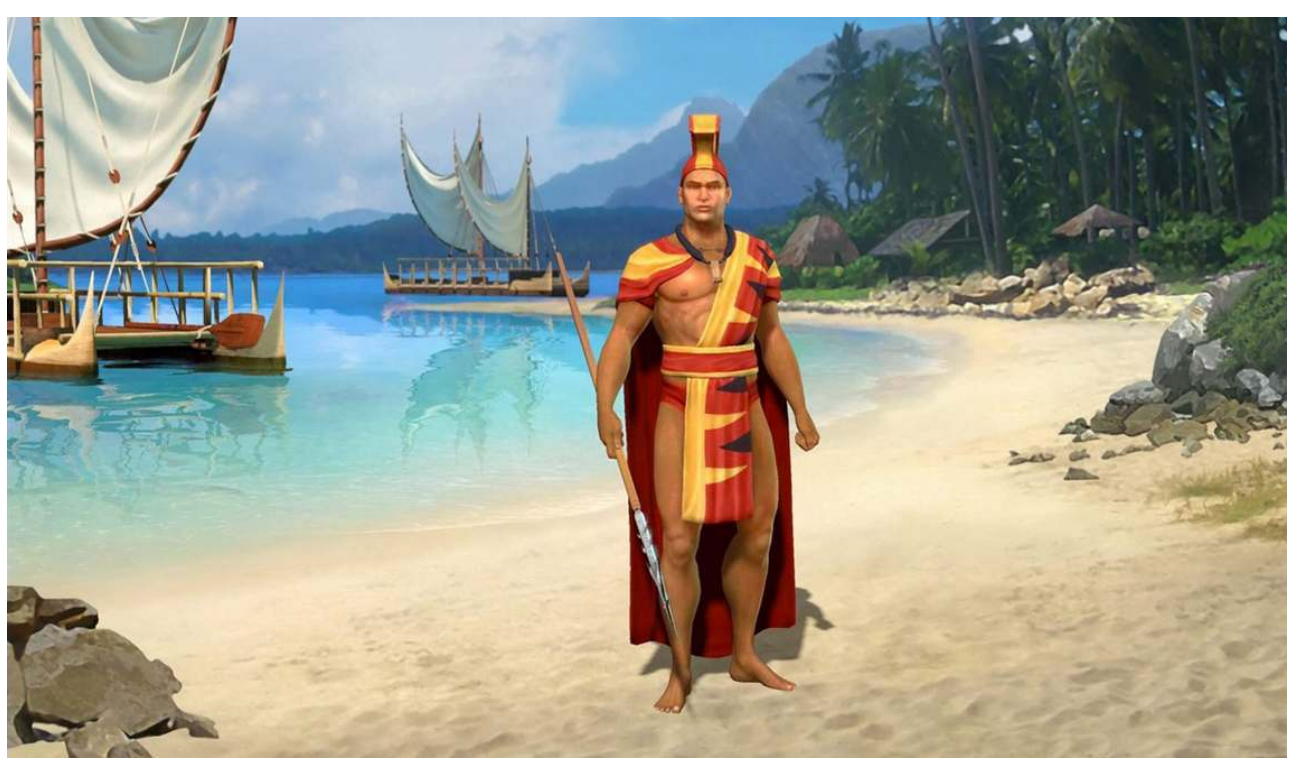

Capture d'écran (2) K Games | Firaxis 
Fig. 4b : Exotisme de l'art maori présent sur l'île d'Hawaï.

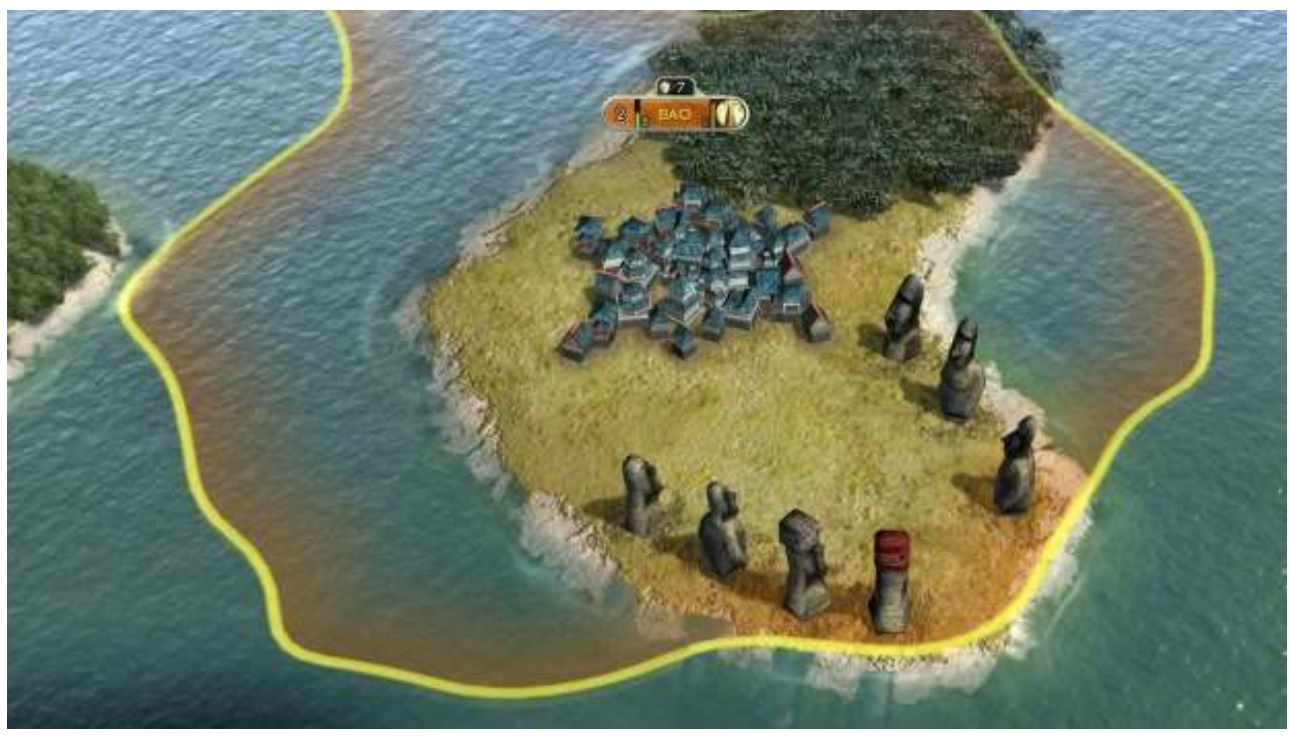

Capture d'écran @2K Games | Firaxis

Dans la continuité de cette première remarque, il est intéressant de constater que figurent en bonne place les "grandes civilisations", celles que l'on attend, qui ont été consacrées par la tradition occidentale et qui sont autant de repères dans le grand récit de l'histoire de l'humanité. La Grèce, Rome, l'Égypte, les Assyriens, les Incas, la Chine, le Japon... La liste ressemble à s'y méprendre aux titres de la série "Grandes Civilisations » parue aux éditions Arthaud dans les années $60^{\prime}$ et $70^{18}$ ou aux volumes de la collection "Les Grandes Civilisations Disparues", éditée à Genève un peu plus tard ${ }^{19}$. Il y a manifestement, dans ce domaine, des classiques indémodables. À côté de celles-ci, figurent cependant dans le jeu, dès 1991, des civilisations plus « originales», comme les Zoulous ou les Songhaï. Cette touche d'exotisme permet, dans les versions les plus récentes, d'enrichir le graphisme, de créer des décors plus variés et des personnages hauts en couleur (fig. $3 a$ et $3 b$ ), qui ont pour effet de surprendre le joueur et d'accentuer l'effet d'uchronie. Il paraît en effet plus intéressant ${ }^{20}$, parce qu'inédit, de faire s'affronter des Romains et des Shoshones, ou les États Unis avec la Polynésie (fig. 4a et $4 \mathrm{~b}$ ), que les Grecs avec les Égyptiens ou les Anglais avec les Allemands.

Dans le processus d'évolution du jeu, on observe en réalité le maintien d'un équilibre soigneusement dosé entre trois types de civilisations: les civilisations traditionnelles (anciennes ou disparues), les États-Nations, saisis dans un moment fondamental de leur histoire, et des civilisations plus "exotiques». Cette répartition équilibrée trahit la volonté des concepteurs d'embrasser l'ensemble des civilisations de l'univers, dans une «histoire » universelle, à la fois mondialisée et transhistorique. L'effet immédiat et apparent de cet élargissement est d'étendre la définition de ce qu'on nomme " civilisation ». Mettre sur le même plan, le temps du jeu, des civilisations considérées comme majeures, comme l'Égypte ou la Chine, et des civilisations moins attendues, comme le royaume de Siam ou le Congo, le devenir des Etats-Unis et celui des Iroquois : l'intention paraît louable. La notice précise en outre qu'aucune civilisation ne constitue un meilleur choix qu'une autre; toutes peuvent mener à la victoire. Ce relativisme proclamé est cependant tellement affiché qu'il en devient caricatural et surtout le descriptif qui accompagne la présentation de chaque civilisation et qui prétend guider le 
joueur dans la sélection de celle qu'il veut diriger, tempère cet égalitarisme en rétablissant clairement des hiérarchies.

Fig. 5 : Askia, le chef de la civilisation Songhai (Civilization V)

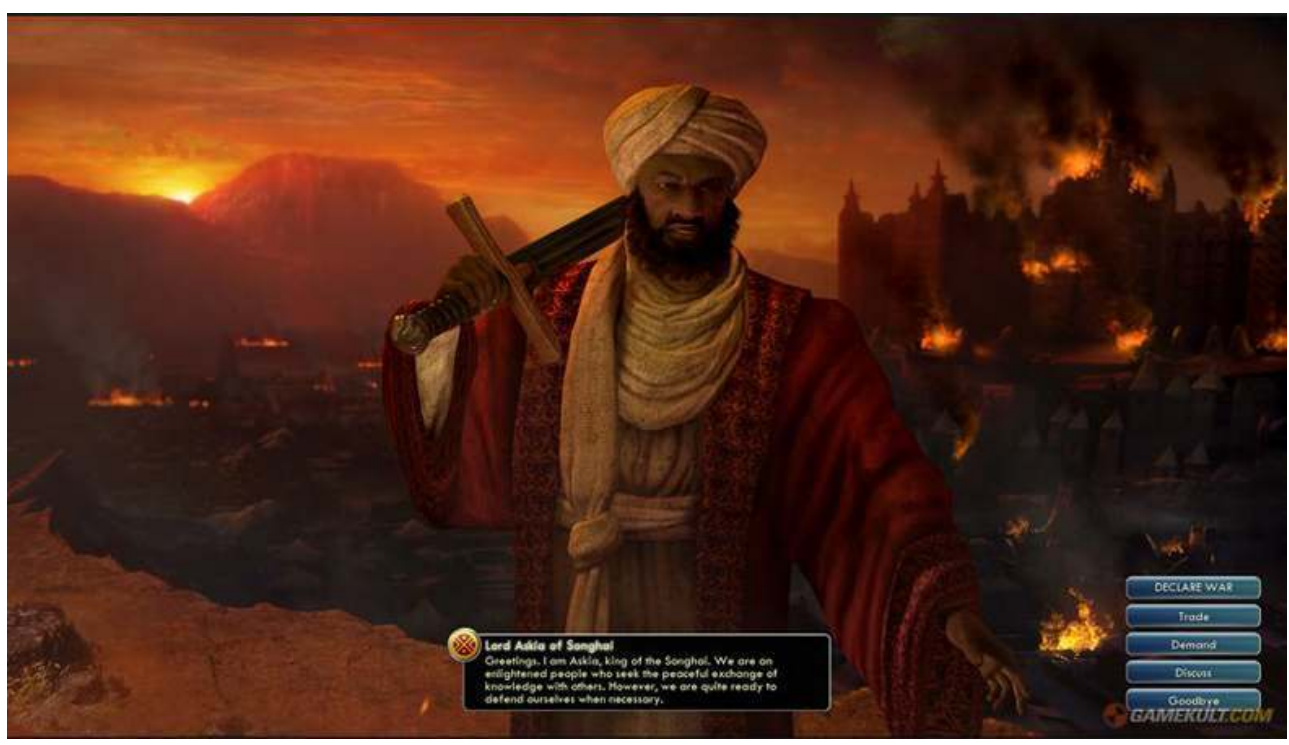

Capture d'écran @2K Games | Firaxis

Dans la description de la civilisation Songhaï (fig. 5), on apprend ainsi que cet empire connut son apogée en Afrique occidentale au $\mathrm{xv}^{\mathrm{e}}$ siècle, autour de sa capitale, Gao, après s'être affranchi de la domination de l'empire du Mali et qu'il s'est étendu pendant deux siècles en devenant «l'un des plus vastes empires de l'histoire africaine ». Cependant, la notice précise aussi que "comme l'Empire aztèque, il est tombé sous les coups d'une bande d'envahisseurs relativement peu nombreux qui disposaient de techniques nettement supérieures ", et elle ajoute : « Ceci est une leçon importante pour tous ceux qui jouent à Civilization : ne te bats jamais avec un couteau contre un fusil. Apporte une arme d'assaut et un bombardier furtif ${ }^{21}$. Cette morale cynique, caractéristique du jeu vidéo $^{22}$, montre aussi l'esprit dans lequel sont proposées les civilisations lointaines : ces civilisations lancent un défi au joueur américain ou européen qui voudra renverser le cours de l'histoire ou se mettre dans la peau du faible pour affronter les " grands $»^{23}$. Qui voudra jouer David contre Goliath.

\section{Dirigeants et dirigeantes : la civilisation et le genre}

Chaque civilisation est menée par un dirigeant (leader) qui lui sert d'emblème. Cette personnification de chaque civilisation a pour double effet de l'associer à une période donnée, présentée comme son apogée, et de la doter de traits spécifiques, qui forment comme son "caractère » (l'équivalent de ce qu'on appelait autrefois «l'esprit » ou le "génie » d'un peuple). Le jeu retient ainsi le personnage le plus représentatif d'une civilisation eu égard à son histoire, un personnage coïncidant avec ce qui est considéré comme sa période d'épanouissement maximal. Comme nous l'avons déjà souligné, la plupart de ces personnages sont des conquérants; ils ont, par leur activité militaire, contribué à l'extension géographique du territoire qu'ils gouvernaient. Napoléon, 
Bismarck, G. Washington, Gengis Khan, Alexandre, Darius $\mathrm{I}^{\mathrm{er}}$ : ces personnages sont des figures attendues.

Fig. 6a : Théodora, impératrice de Byzance (Civilization V)

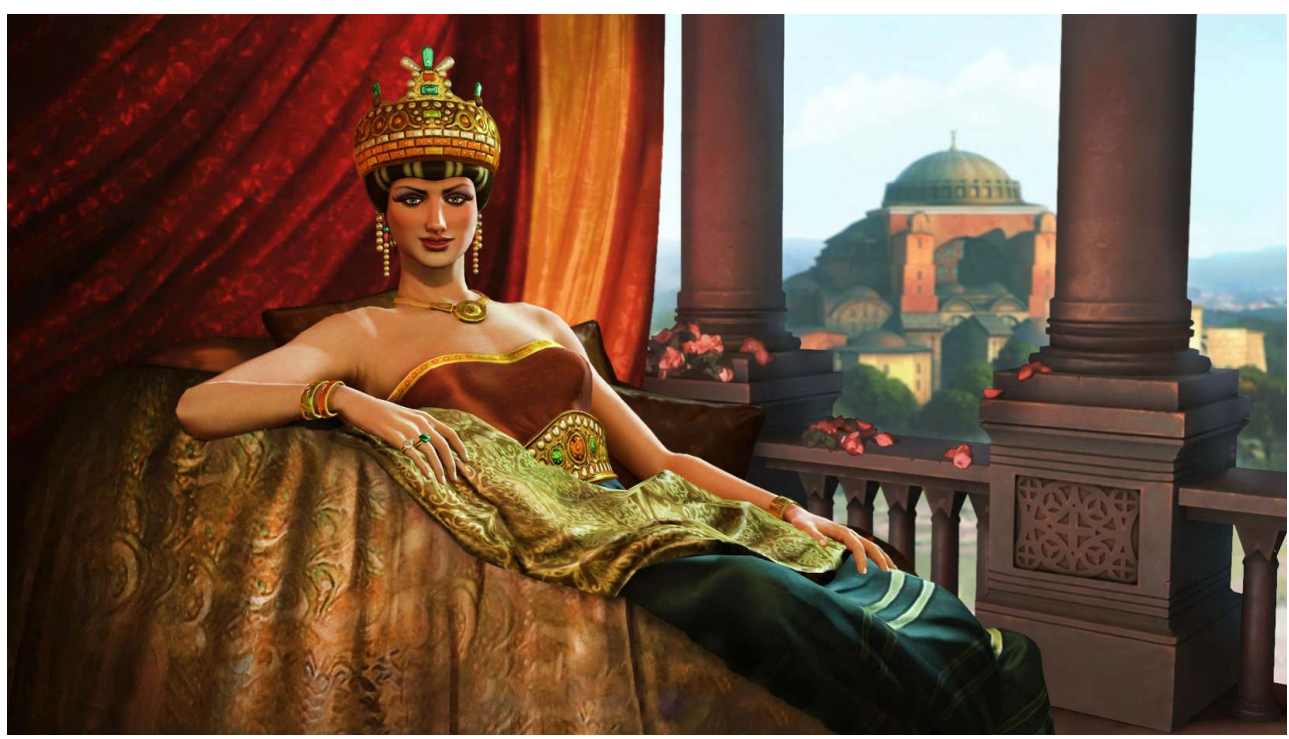

Capture d'écran @2K Games | Firaxis

Fig. $6 \mathrm{~b}$ : Théodora en tenue d'apparat. Détail de mosaïque, Basilique Saint-Vital de Ravenne (547 de notre ère).

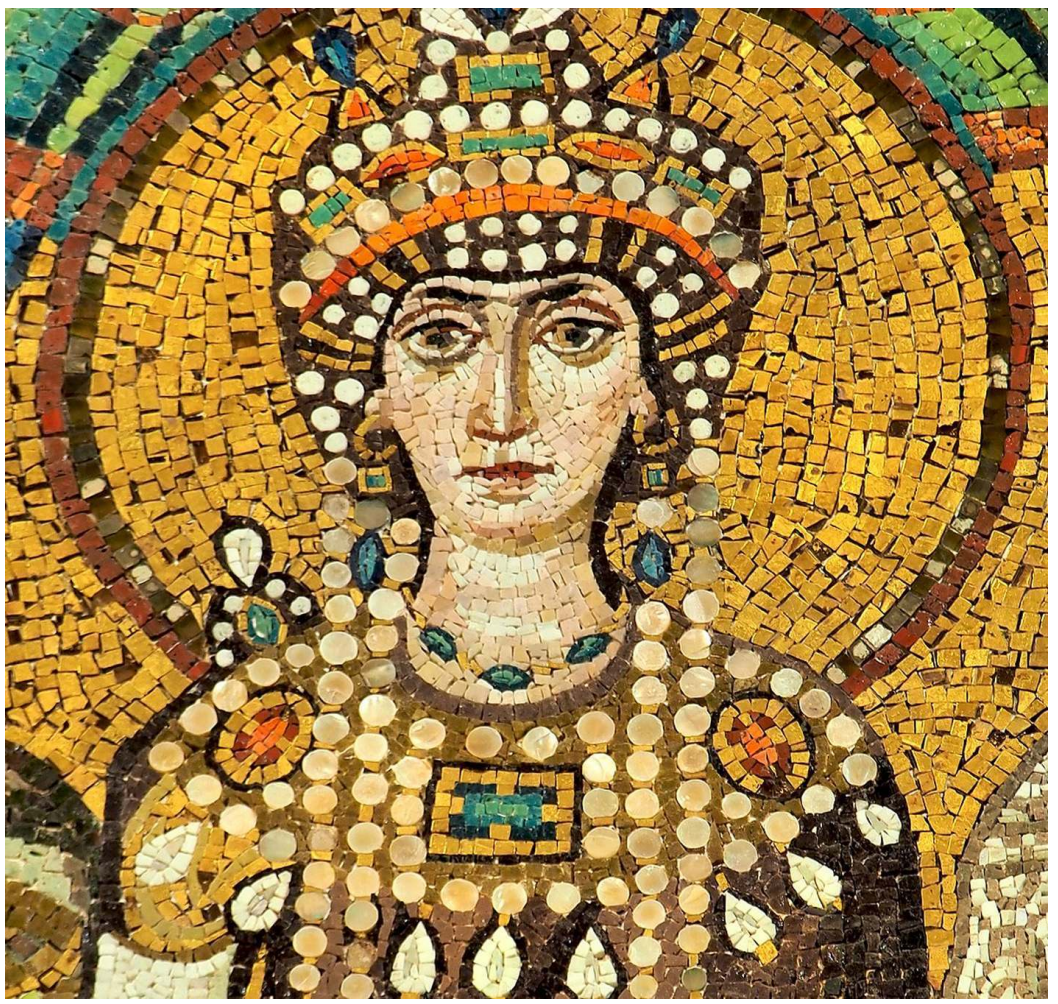


Fig. 7 : Didon, reine de Carthage (Civilization V).

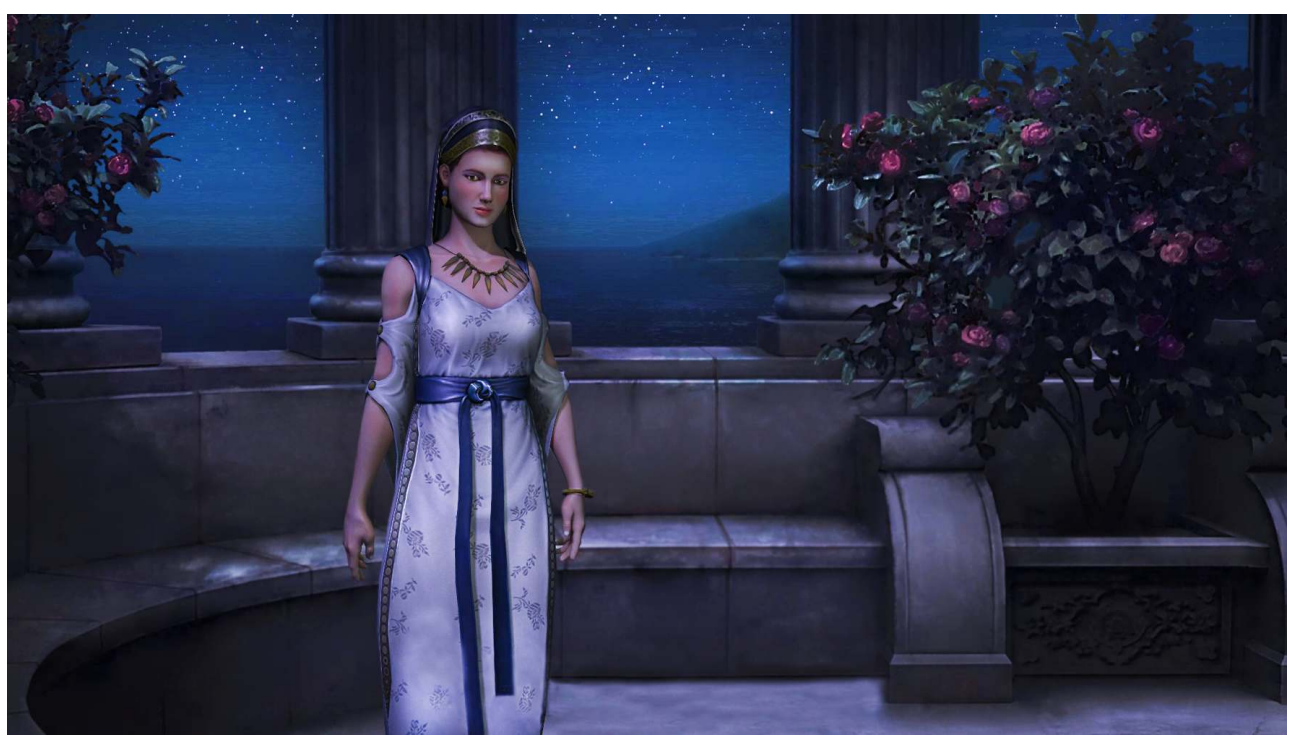

Capture d'écran @2K Games | Firaxis

Il est cependant amusant de constater le rôle donné aux femmes dans cette histoire du monde. Elles y sont relativement nombreuses. Théodora (fig. 6a et 6b), Didon (fig. 7), Isabelle, reine d'Espagne, Victoria, Catherine de Médicis, etc. Cette présence est probablement liée à des enjeux commerciaux: vendre le jeu à un public féminin? permettre aux hommes d'assumer, le temps du jeu, un rôle féminin? Mais le choix de ces dirigeants féminins (female rulers) reflète aussi le poids de certains personnages dans l'imaginaire partagé et le rôle qui leur est attribué dans l'histoire de l'Occident.

Fig. 8 : Elisabeth 1 ère, leader féminin dans Civilization V.

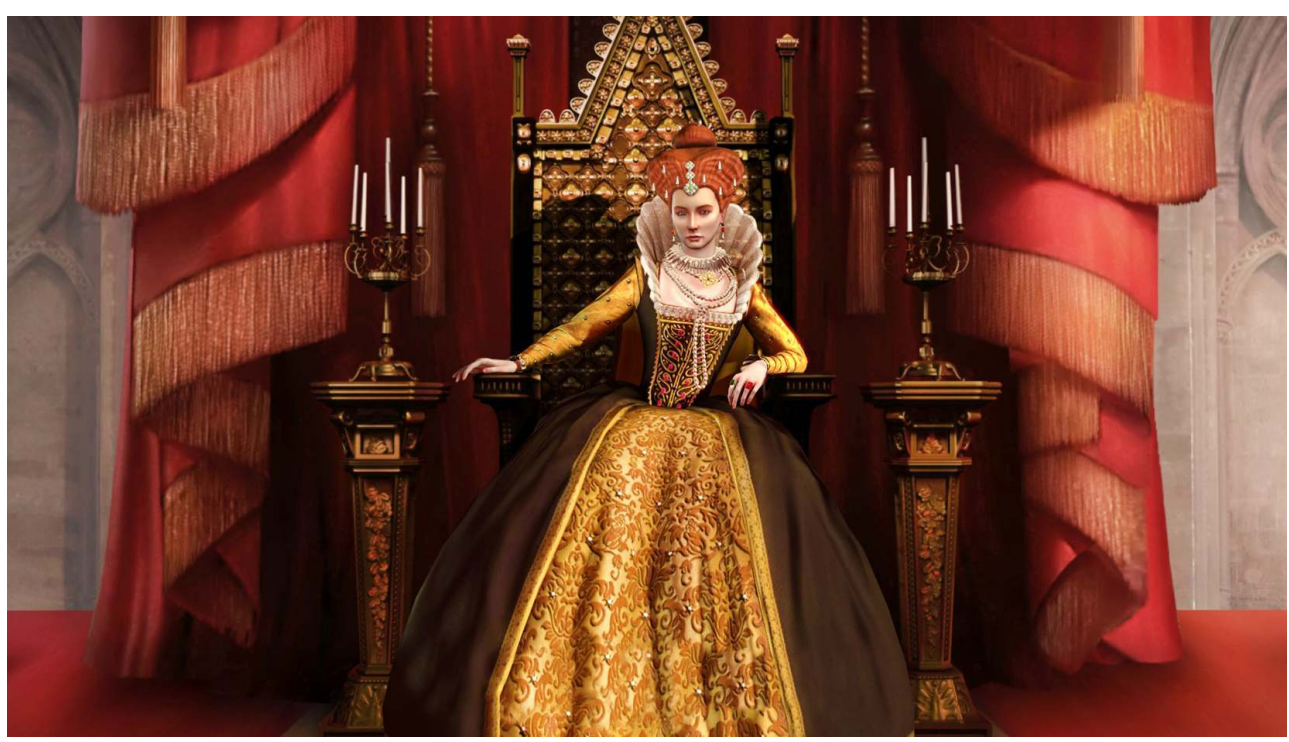

Capture d'écran @2K Games | Firaxis 
Fig. 9 : Cléopâtre, reine d'Égypte (Civilization VI).

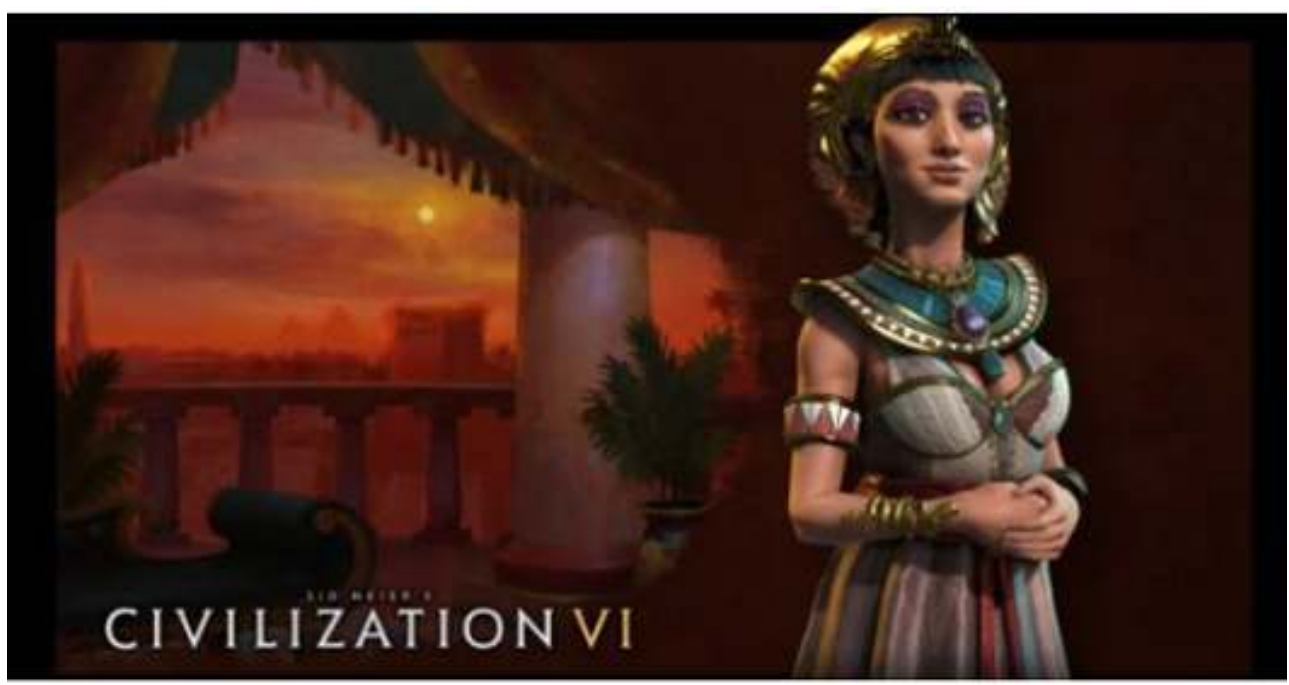

Capture d'écran @2K Games | Firaxis

13 Elizabeth I ${ }^{\text {ère }}$ (fig. 8), Catherine II de Russie ou Cléopâtre (fig. 9) : ces exemples reflètent la célébrité de certaines figures et le relatif consensus à leur égard (Jeanne d'Arc pour représenter la France n'aurait pas eu le même effet). La présence de ces femmes de pouvoir contribue à l'écriture d'une nouvelle histoire du monde dans laquelle l'hégémonie masculine semble à dessein un peu atténuée ${ }^{24}$. Comme les peuples exotiques, les femmes font ainsi partie d'une stratégie de « rééquilibrage » dans une histoire globale et consensuelle, attentive aux minorités.

Fig. 10a : Gandhi, dans Civilization IV, dirigeant de l'Inde, figure pacifique.

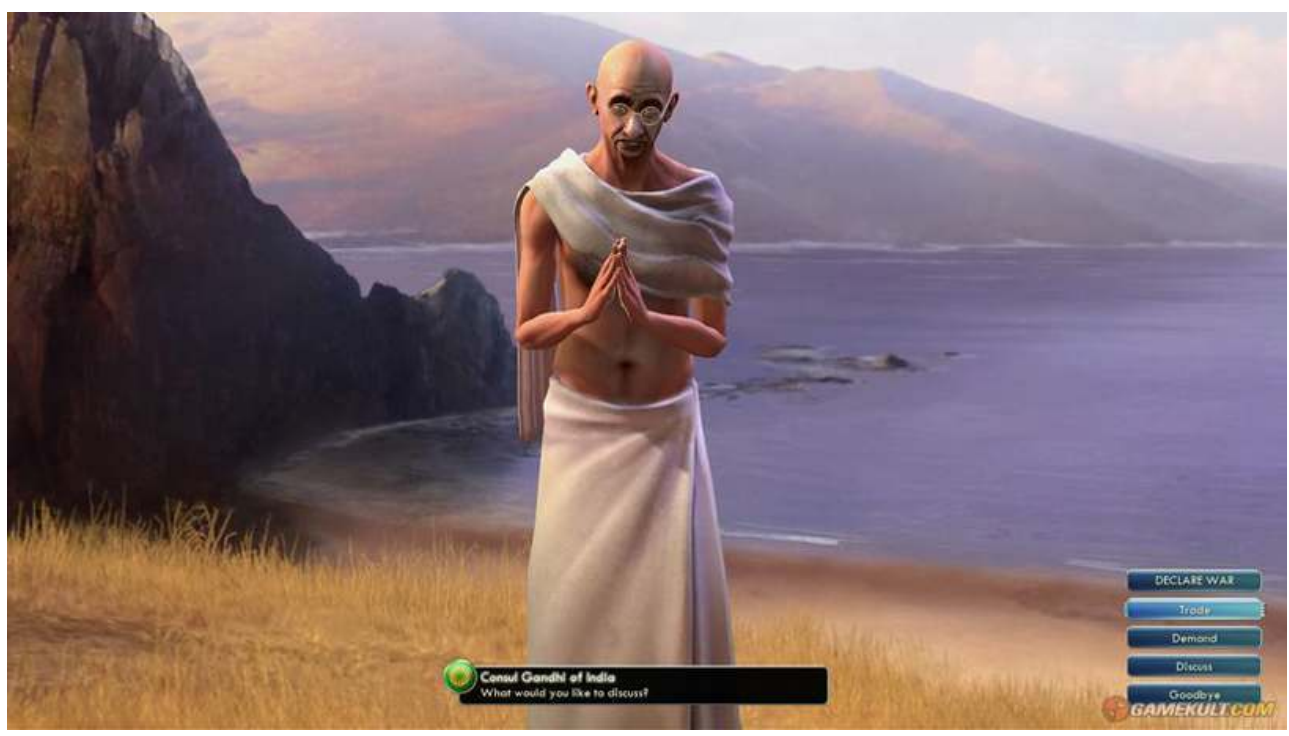

Capture d'écran (C2K Games | Firaxis 
Fig. 10b : « Nuclear Gandhi » : imitation parodique de la couverture de Civilization VI.

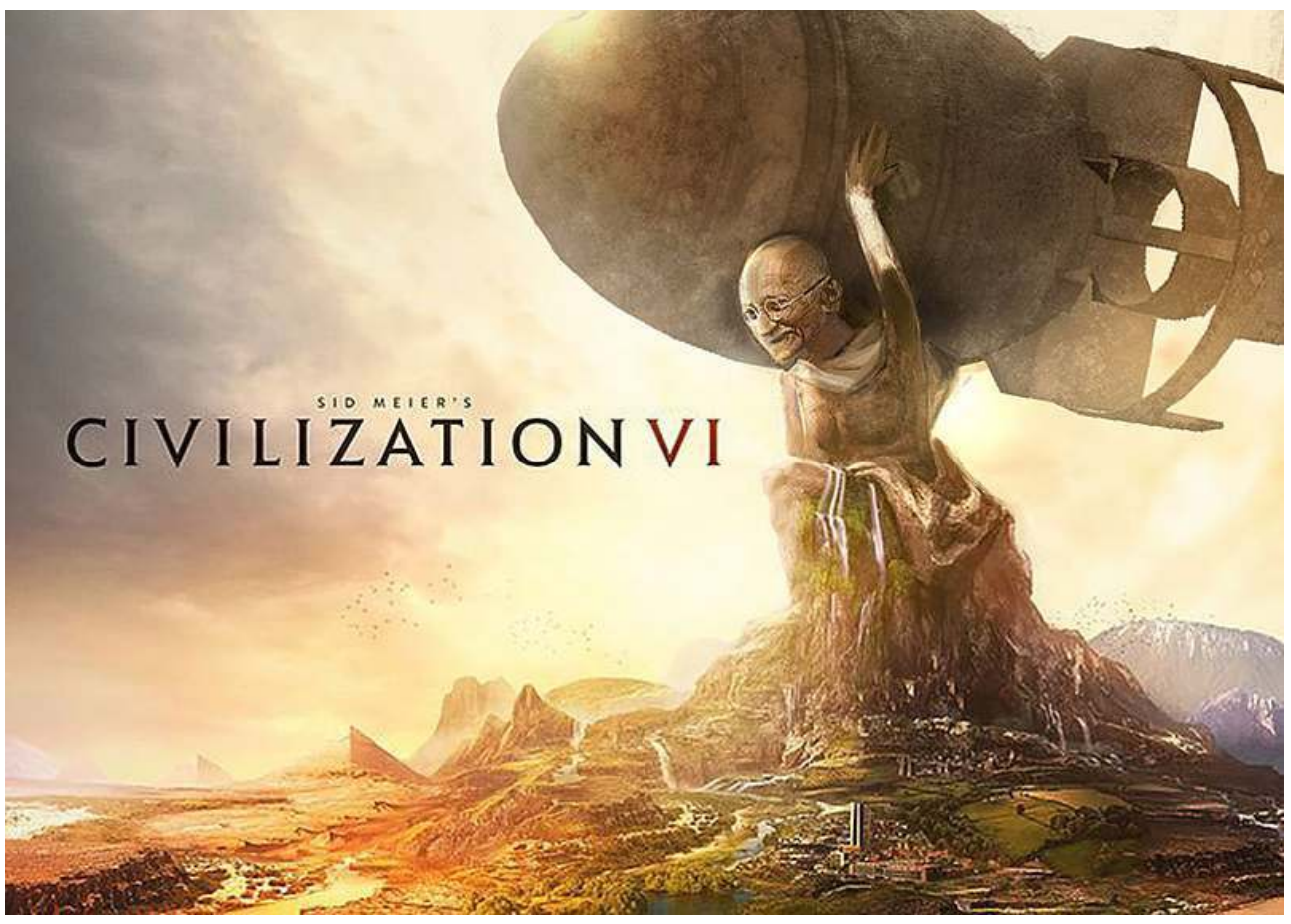

Outre les femmes, on note, parmi les dirigeants, la présence de personnages qui forment le contrepoint de cette histoire conquérante. Civilization a par exemple fait le choix de Mohandas Karamchand Gandhi pour mener l'Inde (fig.10a). Ce chef, doté de « spiritualité » et de " philosophie $»^{25}$, permet ainsi aux joueurs pacifistes de trouver leur bonheur dans le jeu; il est inutile de choisir ce personnage (et donc cette « civilisation » qu'est l'Inde) si vous voulez l'emporter par les armes. Le jeu joue donc avec les stéréotypes. Il les utilise, les affirme et même les accuse à dessein. Mais parfois aussi, il les détourne. Au point que dans la version $\mathrm{V}$ du jeu, le personnage de Gandhi est doté d'une grande « agressivité » et apparaît comme le meilleur choix pour mener des combats sans merci ${ }^{26}$ (fig. 10b). Ce simple procédé d'inversion est une façon de fabriquer du second degré à peu de frais, tout en continuant à véhiculer les mêmes stéréotypes.

\section{L'urbanisme, signe de civilisation}

Dotée d'un chef et de certains "traits de caractère ", une civilisation doit également posséder un territoire. Amené à gérer un espace géographique ${ }^{27}$ très limité en début de partie, le joueur a pour mission d'accroître progressivement ${ }^{28}$ et surtout d'aménager ce territoire, de façon à augmenter sa population. Pour cela, il doit d'abord construire des villes, puis explorer les territoires qui les entourent, enfin occuper, aménager et défendre les espaces ainsi conquis. Le rôle fondamental donné aux villes dans le jeu Civilization est très représentatif de la conception occidentale contemporaine de ce qu'est une civilisation. L'urbanisation croissante est vue comme un signe de développement nécessaire. Il est impossible de gagner sans cités puissantes et bien situées. Le joueur est donc invité à bâtir le plus vite possible une ville qui deviendra la capitale de sa civilisation; sa population croit au fur et à mesure de la construction et de l'aménagement de ses villes, qui sont des lieux de production de richesses ${ }^{29}$. Le jeu révèle 
en outre une vision très normée de ce que doit être une ville, à la fois en termes d'espace et en termes démographiques.

Fig. 11a : Un plan hippodamien : construction de ville dans Civilization III.

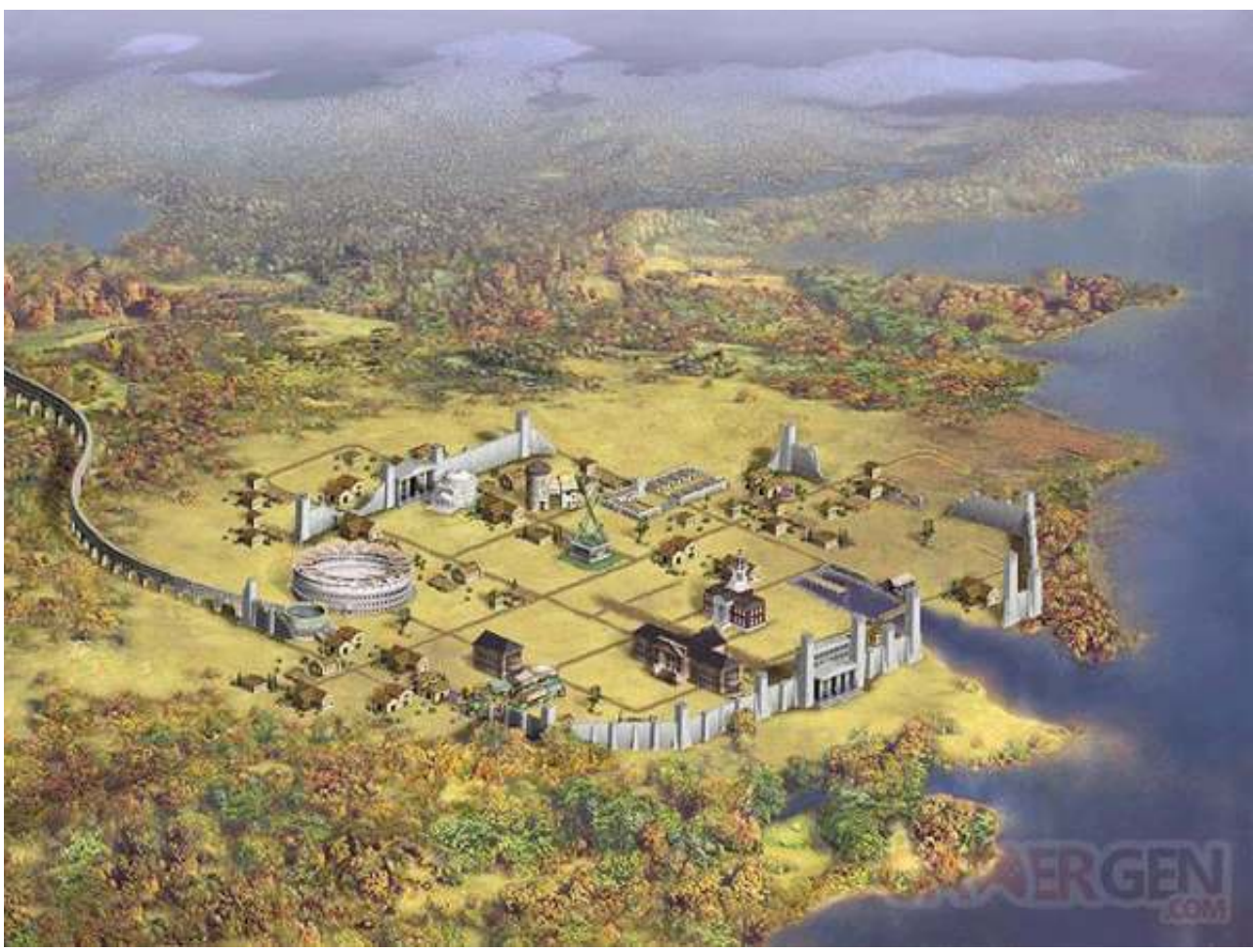

Capture d'écran @2K Games | Firaxis

Fig. 11b : Un lieu saint fait d'édifices de style différents, une plateforme commerciale et une zone industrielle autour de Yokohama. Spécialisation par quartiers dans Civilization VI.

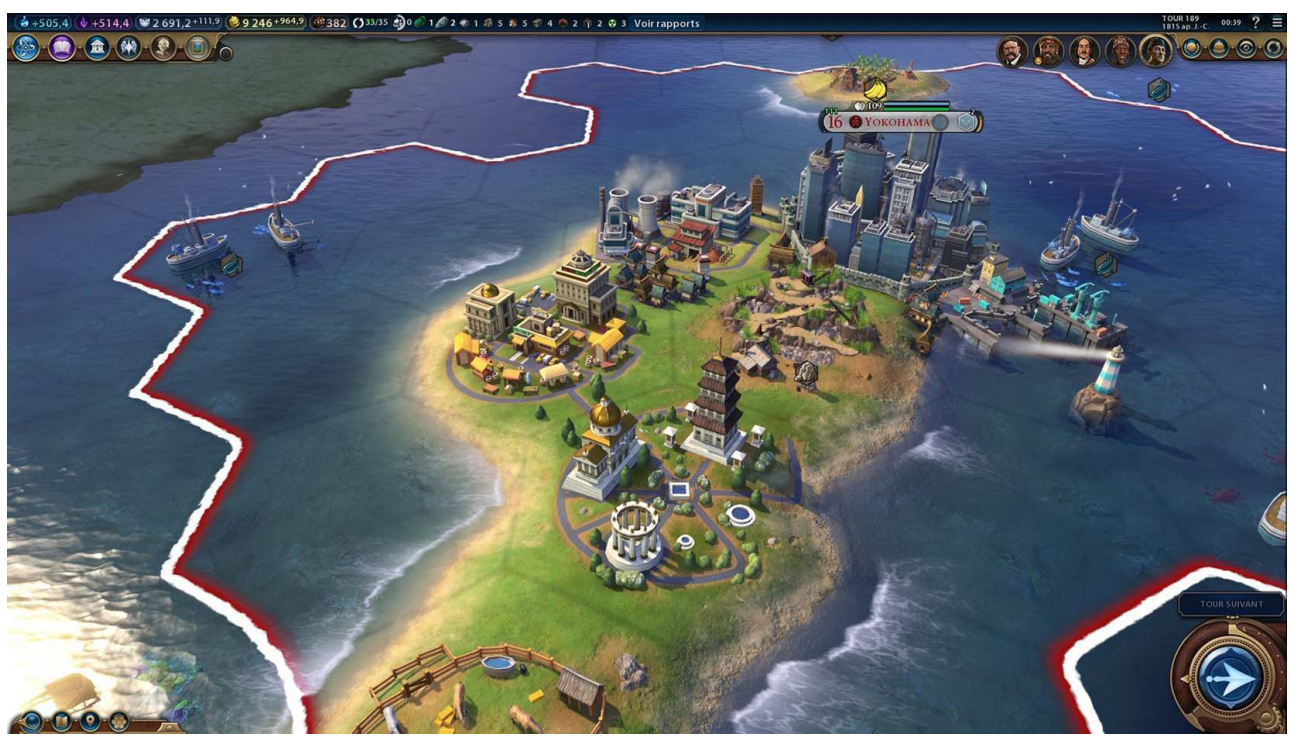

Capture d'écran @2K Games | Firaxis

16 Aménagée à proximité d'un cours d'eau, dans une zone de plaine, son plan est conforme à la logique urbanistique des villes américaines: un plan hippodamien (fig. 11a), une 
division en quartiers spécialisés, la présence de bâtiments spécifiques et standardisés ${ }^{30}$. Outre les habitations, la ville doit comporter un certain nombre d'infrastructures, écoles et bibliothèques, marchés et greniers, banques et casernes, qui répondent chacune à une fonction bien définie (fig. 11b). Le scénario du jeu repose enfin sur l'idée qu'une ville trop peu peuplée n'aura pas de forces vives pour résister aux assauts des civilisations concurrentes (le village constitué de quelques huttes ne peut survivre longtemps), mais qu'il est également nécessaire de garder une certaine mesure: une démographie trop forte crée du "Mécontentement " (Unhappiness) et risque de provoquer des révolutions ${ }^{31}$. Il est facile de reconnaître, dans cette conception, le préjugé négatif associé aux villes densément peuplées des pays émergents. Urbaine, disposant d'un territoire étendu, une civilisation doit enfin exercer une influence sur le reste du monde ${ }^{32}$. L'autarcie ou le repliement sur soi n'est pas une idéologie transmise par le jeu Civilization.

\section{Les barbares contre la civilisation}

Mais la civilisation se définit aussi par ce qui s'oppose radicalement à elle : les barbares ${ }^{33}$. Ces personnages jouent un rôle important dans le jeu. Sans nom, les «barbares » sont des ennemis et n'ont d'autre fonction que de s'opposer à la progression des civilisations, de toute civilisation ${ }^{34}$. Il faut donc les combattre et les anéantir, et ce, dès le début du jeu et il n'y a, pour y parvenir, pas d'autre moyen que de faire et de remporter la guerre ${ }^{35}$, car les barbares ne connaissent pas la diplomatie ${ }^{36}$. Dans les premières éditions du jeu, ils n'apparaissaient qu'au début et étaient donc assez faciles à éliminer. Dans les éditions plus récentes ${ }^{37}$, les barbares peuvent s'équiper de technologies militaires qui les rendent nettement plus coriaces. Ils gardent cependant une altérité et un statut qui les opposent radicalement aux civilisations.

Fig. 12 : Barbare dans Civilization VI (Revolution).

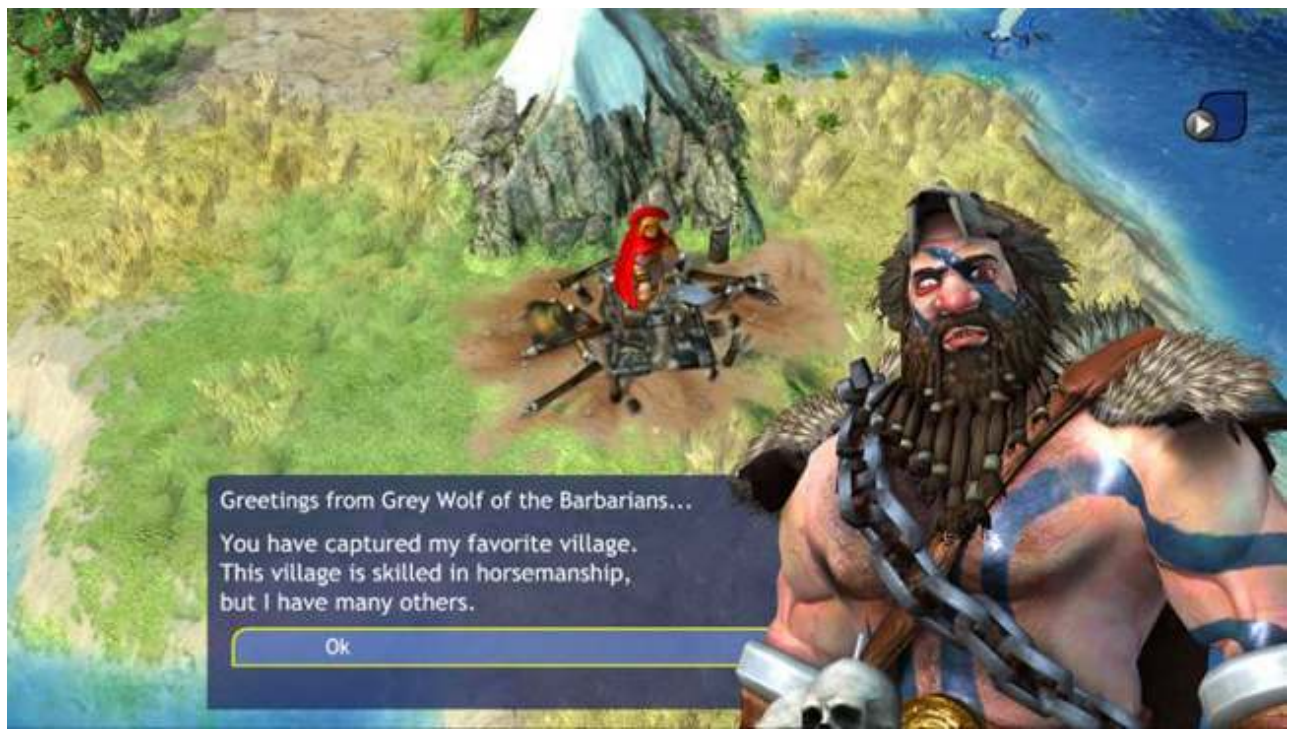

Capture d'écran @2K Games | Firaxis 
Fig. 13 : Menace barbare matérialisée à l'écran par des personnages à demi-nus armés de gourdins.

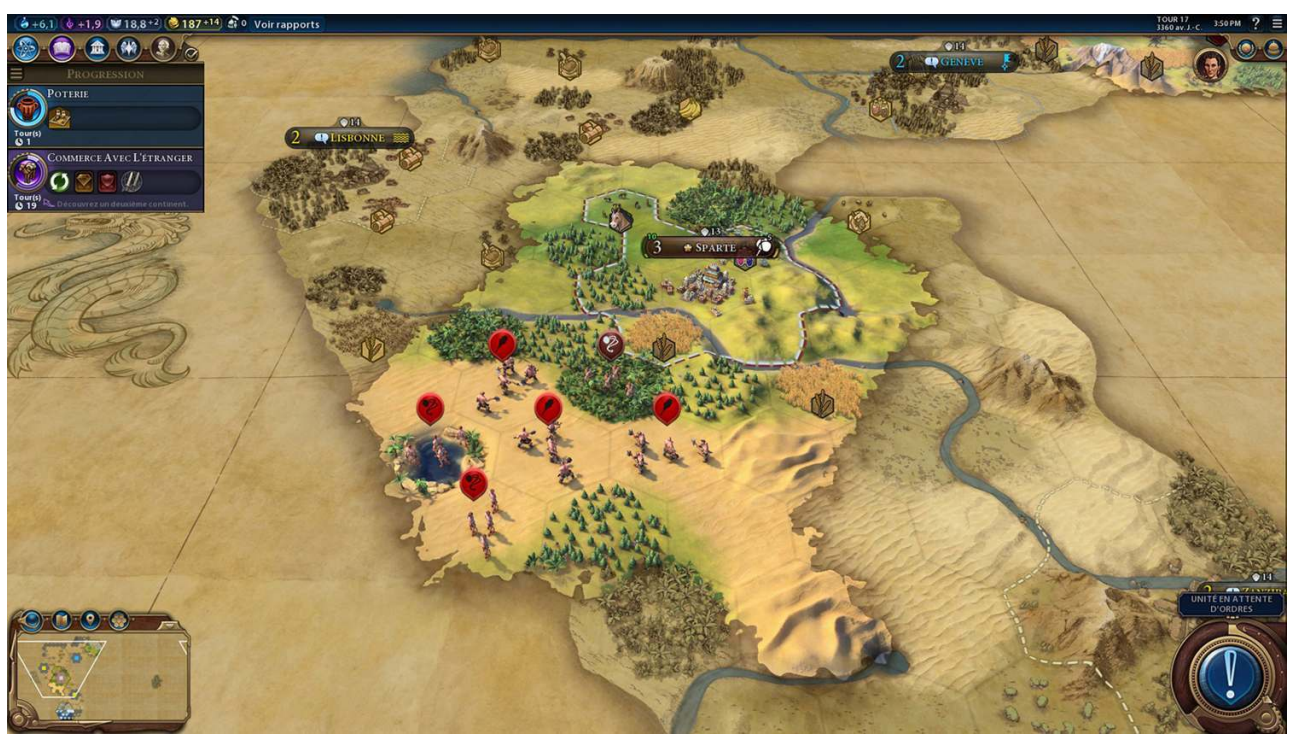

Capture d'écran @2K Games | Firaxis

Le graphisme et l'environnement sonore mettent en évidence leur sauvagerie (fig. 12). Chevelus, à demi nus, les barbares crient indistinctement ${ }^{38}$, ils vivent en bandes dans des campements (fig. 13), souvent dissimulés et ont un mode d'agir spécifique : ils attaquent systématiquement toutes les unités qu'ils rencontrent, se livrent au pillage, créent du désordre. Mais plus la civilisation gagne de terrain, moins les barbares auront de place et donc de pouvoir de nuisance ${ }^{39}$ et surtout toute victoire remportée par les barbares affaiblit, voire peut détruire une civilisation, mais jamais elle ne leur permettra de former une civilisation nouvelle.

\section{Vie, mort et progrès des civilisations}

Les barbares, par la menace qu'ils représentent, contribuent cependant à renforcer ce qu'est une civilisation, car le propre d'une civilisation est de mourir un jour. C'est en effet ce qu'affirment, en creux, les concepteurs du jeu. Tout d'abord, la fin de partie signe, la plupart du temps ${ }^{40}$, la disparition d'une ou plusieurs civilisations. Ce rapport au temps est d'ailleurs explicité dans les lignes qui présentent le principe du jeu. «Grand dirigeant, votre peuple compte sur vous pour le guider. Saurez-vous le mener vers la grandeur et construire un empire qui passera l'épreuve du temps ${ }^{41}$ ?» L'idée que chaque civilisation connaît un début, un moment d'apogée, puis une fin inéluctable est sans cesse répétée dans le guide accompagnant le jeu. Cette conception apparait aussi en filigrane dans le rôle attribué aux ruines.

Les ruines sont en effet des éléments présents dans l'espace de jeu virtuel; elles ne sont pas immédiatement visibles, mais les joueurs ont tout intérêt à les découvrir car elles apportent des bonus ${ }^{42}$ à ceux qui en prennent possession. Ces ruines sont présentées comme les restes de civilisations passées ${ }^{43}$. Autrement dit, si le jeu commence à l'aube des civilisations, il postule aussi l'existence d'un passé encore plus ancien, un temps «préludique " comme on dit "préhistorique ", dont témoignent les vestiges de civilisations disparues. Le rôle assigné aux ruines dans le scénario sous-entend donc d'une part, que 
les civilisations sont mortelles, d'autre part, qu'elles laissent des traces dans le présent et enfin, que la découverte et la mainmise sur ces vestiges accroissent la richesse et la puissance des civilisations qui s'en emparent ${ }^{44}$. Le temps de la civilisation est un temps linéaire et cumulatif.

C'est aussi la linéarité et la logique cumulative qui déterminent le rôle assigné aux technologies dans le développement des civilisations. Une explication présente dans l'encyclopédie en ligne dédiée au jeu résume la philosophie de l'ensemble: "L'avancée technologique rend la civilisation plus forte, plus grande, plus intelligente et elle renforce sa résistance face à l'adversaire. Il est absolument nécessaire pour une civilisation de maintenir son niveau technologique par rapport à ses voisins ${ }^{45}$. 》

Fig. 14 : L'arbre des technologies (Civilization V).

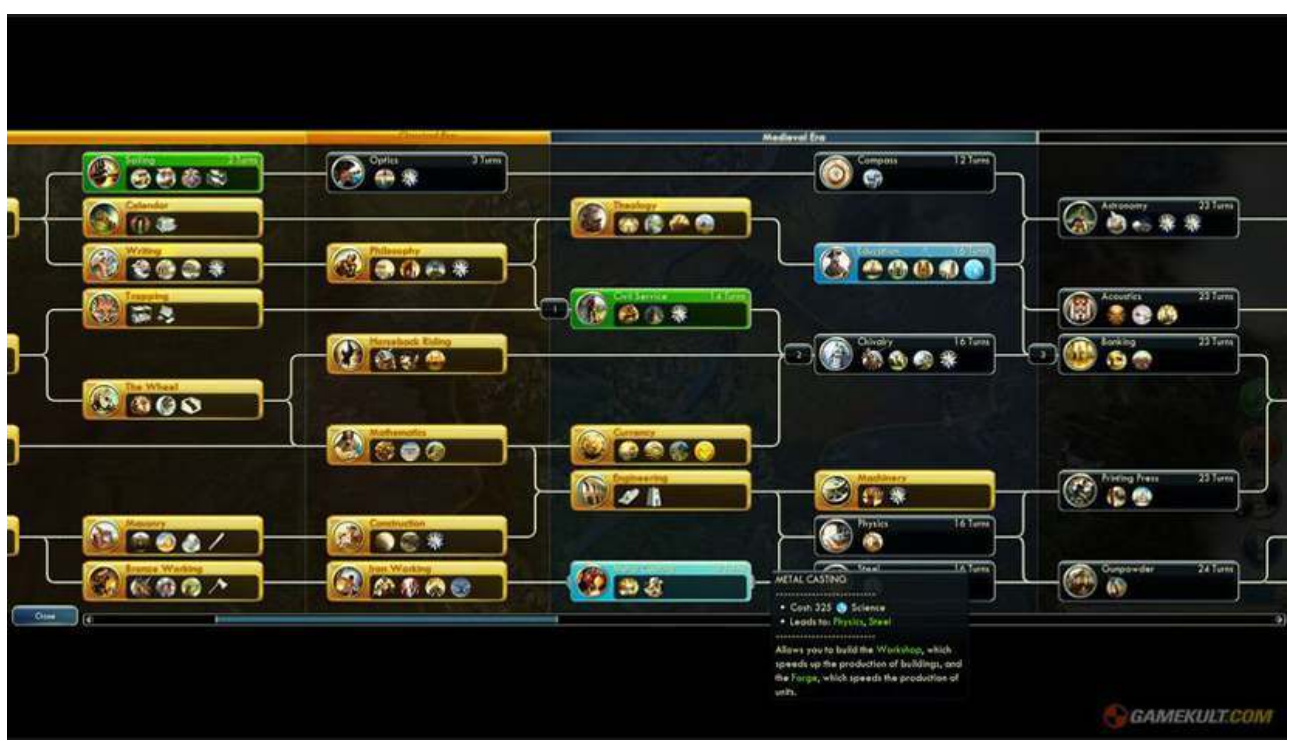

Capture d'écran @2K Games | Firaxis

Civilization témoigne en effet d'une vision du progrès technique érigé en dogme, dont l'arbre des techniques (technology tree) fournit une bonne illustration (fig. 14). Cet élément-clé du jeu, affiné dans chaque version, est un schéma exprimant graphiquement le chemin qui conduit chaque civilisation de l'origine jusqu'à l'ère moderne. Présenté de manière horizontale et se lisant de gauche à droite, l'arbre des techniques vise à mettre en évidence l'ordre d'acquisition de chaque technologie et le moyen le plus court pour y $\operatorname{arriver}^{46}$ : le joueur est contraint, à la fois, par une logique chronologique, puisque chaque technique est associée à une ère spécifique (on ne peut pas acquérir le compas avant le Moyen Âge ${ }^{47}$ et il faudra attendre l'Ère industrielle pour accéder à la dynamite) et par une logique de type généalogique et combinatoire, puisqu'il est souvent nécessaire de posséder deux techniques préalables pour en acquérir une troisième. Il est ainsi indispensable d'avoir les mathématiques et la construction avant de pouvoir " trouver " l'ingénierie. Ce schéma est logique : il correspond en grande partie à la chronologie réelle des différents types d'inventions. Mais il contient aussi une part d'arbitraire, qui maintient le joueur dans un espace ludique ${ }^{48}$. L'arbre technologique rend aussi manifeste l'idée que certaines «inventions » sont plus nécessaires que d'autres. L'agriculture par exemple est présentée comme l'élément premier de toute civilisation ${ }^{49}$ et l'écriture comme une étape obligée. 
23 Si toutes les civilisations suivent le même processus de développement, tout aussi explicite est l'idée qu'elles n'avancent pas au même rythme. Toute la stratégie repose en effet sur la vitesse d'acquisition des différentes techniques, qui peuvent s'obtenir de façons diverses, mais dont la possession conditionne les progrès dans le jeu, et donc la victoire finale. Le scénario met en évidence les différences de niveau de développement à un certain moment du jeu: certaines civilisations seront plus "avancées", d'autres moins. Il n'y a, dans ces différences de tempo, aucun déterminisme ; c'est principalement l'action du joueur (son agentivité, agency) qui aura des effets sur le cours de l'histoire mondiale. Ainsi, les Iroquois pourront, s'ils savent profiter de leurs atouts spécifiques, accéder rapidement aux techniques (construction, travail du métal...) qui leur permettront de construire la Tour Eiffel ou de lancer le Projet Apollo depuis leur capitale.

Les notices consacrées à chacune de ces technologies rappellent parfois quel est l'inventeur historique de telle ou telle. Le jeu attribue ainsi aux Égyptiens l'invention du premier calendrier, aux Chinois, la poudre à canon ; la plus ancienne mine est associée au Swaziland, tandis que les Scythes nomades sont les ancêtres des cavaliers. Mais l'essentiel est de comprendre que toutes ces techniques sont liées au processus de civilisation, qu'elles sont apparues de façon progressive, que certaines civilisations les ont "inventées", puis diffusées ou échangées, et que le monde moderne, celui des civilisations avancées, s'appuie sur cet héritage commun pour continuer à en créer, dans une sorte de processus continu et illimité.

\section{Les grands hommes au cœur de l'histoire}

Inséparable des technologies, un autre élément remarquable de Civilization est le rôle attribué aux Grands Hommes (Great People). En effet, l'un des moyens pour favoriser le développement d'une civilisation, accéder à de nouvelles technologies, construire de nouveaux bâtiments, accumuler des points de culture, des richesses supplémentaires, ou même l'emporter dans un combat est de créer un ou plusieurs Grands Hommes, dans l'une des catégories suivantes: marchands, artistes, savants, ingénieurs ou généraux ${ }^{50}$. Ces Grands Hommes ont des noms : parmi les artistes, Mozart et Shakespeare voisinent avec Léonard de Vinci ; A. Carnegie, le philanthrope de l'acier, fait partie des ingénieurs illustres avec G. Eiffel, tandis que Robert E. Lee se retrouve avec Épaminondas ou Scipion l'Africain dans la liste des grands généraux. Pour la plupart, ces noms font donc référence aux personnages les plus célèbres de la culture occidentale ${ }^{51}$, mais le jeu permet de les associer à n'importe quelle civilisation. S'il dirige la Chine, le joueur peut ainsi choisir de faire bénéficier l'Empire du Soleil Levant du talent de Paul Gauguin ou de Lewis Carroll ; Elvis peut naître à Berlin ou Copernic à Los Angeles. Ces « aberrations » montrent que ces dénominations fonctionnent comme de simples icônes; elles personnifient la perfection musicale ou le talent pictural, la science ou l'art du commerce, elles ne désignent jamais le personnage historique. À tel point d'ailleurs que le joueur peut même, s'il le souhaite, donner un autre nom à son Grand Homme ${ }^{52}$.

L'existence de ce type de personnages et surtout le pouvoir qui leur est donné d'accélérer le cours de l'histoire, de « booster » la civilisation, pour reprendre le terme anglo-saxon, s'appuient sur l'idée que l'histoire des civilisations est liée à l'émergence de personnalités exceptionnelles dont l'action ou l'œuvre ont contribué à accroître la renommée du peuple qui les a vus naître. Cette idée n'est pas neuve. Le culte des Grands hommes, très important, notamment en France, à la fin du $\mathrm{XVIII}^{\mathrm{e}}$ siècle $^{53}$, apparaît également comme 
une caractéristique de l'écriture de l'histoire occidentale au $\mathrm{XIX}^{\mathrm{e}}$ siècle ${ }^{54}$. Le jeu vidéo continue de transmettre (ou plutôt réactive) cette vision de l'histoire en l'adaptant à un contexte mondialisé où les «talents » se transportent d'une civilisation à l'autre. On peut en effet, en prenant une ville adverse, se saisir d'un Grand Homme et profiter de ses apports. C'est une version ludique de la « fuite des cerveaux », mécanisme bien connu des États Unis et qui contribue largement à asseoir sa puissance.

\section{L'Antiquité dans l'univers vidéo-ludique de Civilization}

Si l'on a pu apercevoir quelques-uns des éléments qui «font la civilisation », on peut maintenant tenter d'analyser quelle place est attribuée, dans ce jeu, à l'Antiquité et voir quels traits spécifiques ont été retenus et mis en avant pour faire de la Grèce et de Rome des « civilisations ».

\section{"L'ère » antique}

Fig. 15 :« Bienvenue dans l'Ere Classique ! » Ecran d'accueil signalant l'entrée du joueur dans une nouvelle ère (Civilization $V$ ).

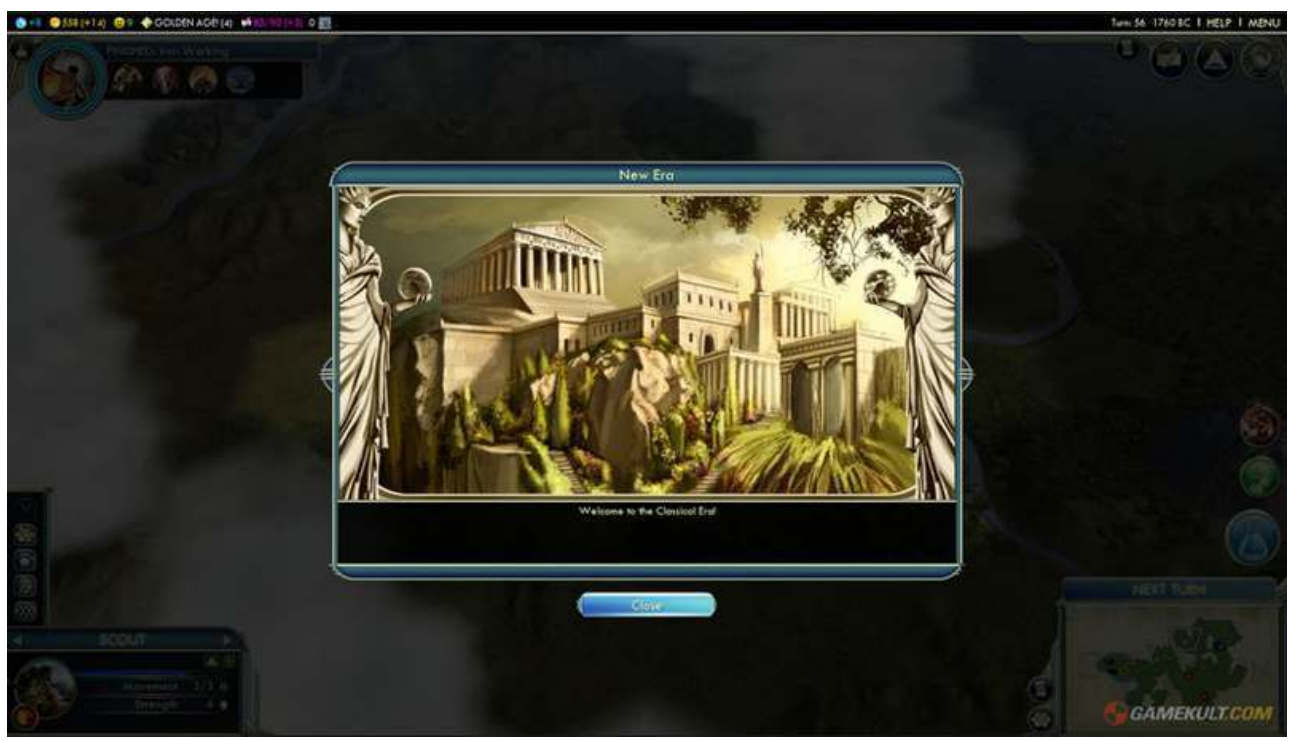

Capture d'écran @2K Games | Firaxis 
Fig. 16 : Le « brouillard de l'inconnu ».

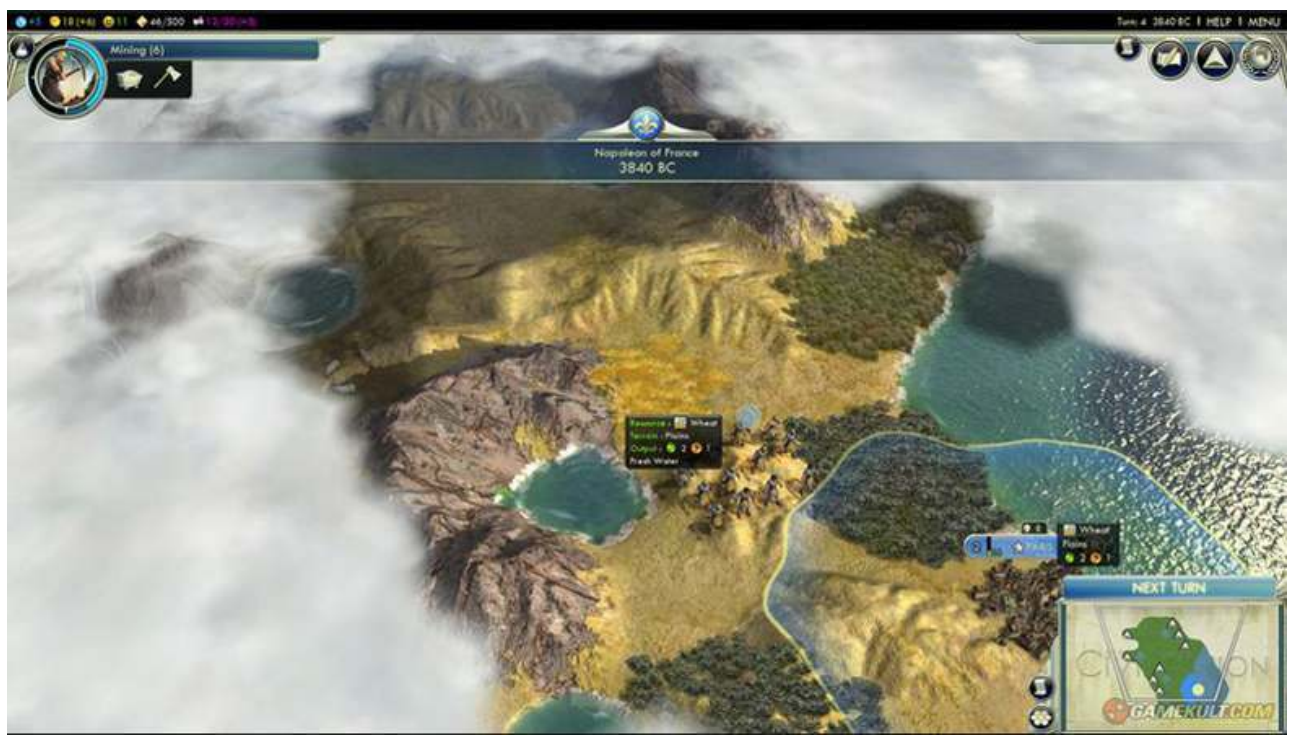

Capture d'écran @2K Games | Firaxis

Précisons immédiatement que «l'Antiquité » a dans le jeu un statut ambivalent. Le mot fait d'abord référence à un moment du jeu, une "ère ", placée au tout début. Ou plutôt elle englobe deux ères distinctes puisque le Moyen Âge est précédé des Temps anciens ( Ancient Era) et du Classicisme (Classical Era) (fig. 15). Ces deux dénominations, rappelonsle, ne renvoient pas à une époque précise, mais se situent dans un temps relatif, celui du $j^{j e u^{55}}$. Ce temps est celui des commencements. Peu de technologies sont à disposition. Les barbares sont menaçants; il faut les débusquer pour les éliminer. Le joueur consacre l'essentiel de son énergie à explorer un territoire qu'il ne connaît pas encore: la manifestation visible de cette méconnaissance et de cette exploration se matérialise à l'écran par un brouillard qui se dissipe progressivement (fig. 16). Passer les montagnes pour voir ce qu'elles cachent, défricher les terrains insalubres (forêts, marais), choisir un endroit propice à l'établissement d'une première ville: cette première étape est commune à l'ensemble des civilisations. L'«aube des civilisations » est un moment de fondation, celui des premiers déplacements, de la confrontation avec l'inconnu, des premières rencontres avec les autres. Les possibilités de jeu restent cependant encore très limitées. Au point même que la dernière édition propose un mode accéléré permettant de «sauter» l'Ère Antique et même le Moyen Âge pour aller directement dans l'Âge moderne ! Joueurs pressés, « l'Antiquité » n'est pas pour vous ! 
Fig. 17 : Ramsès II, dirigeant de l'Egypte dans Civilization V.

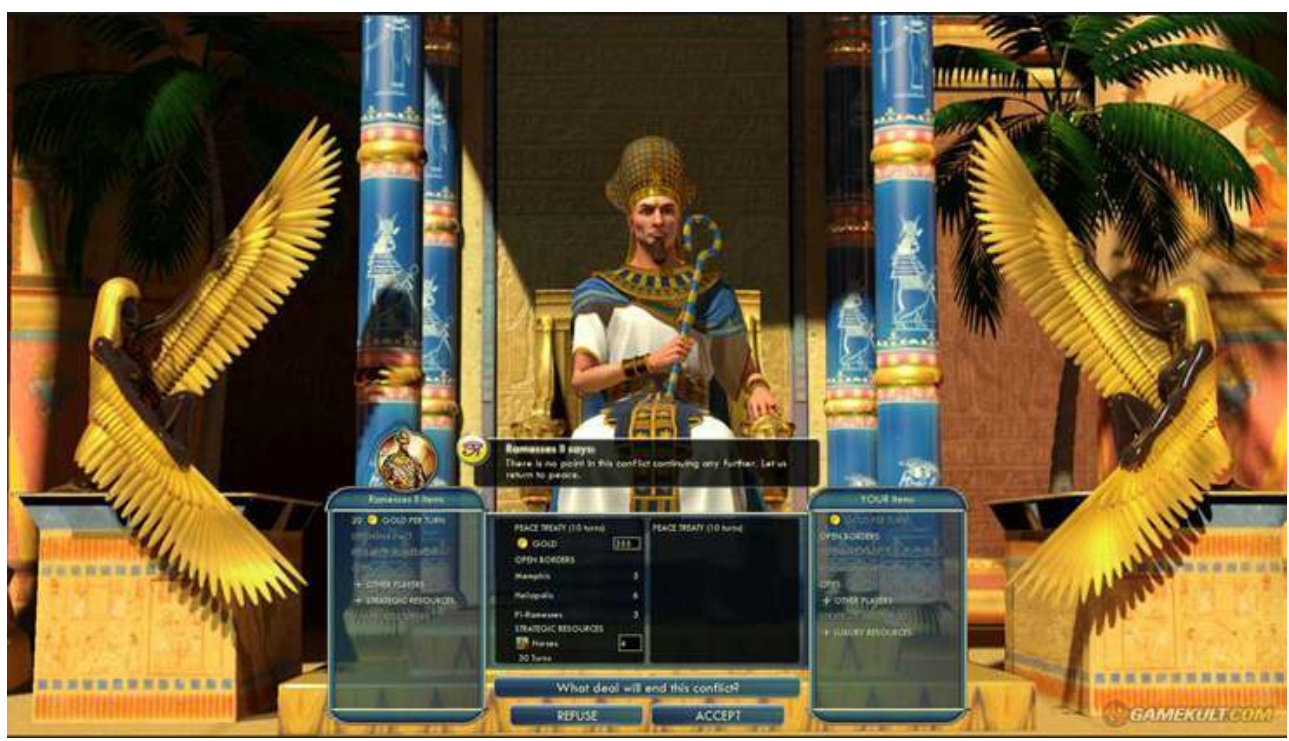

Capture d'écran @2K Games | Firaxis

Nom générique pour désigner le temps du commencement, l'Antiquité est également présente à travers un éventail de civilisations qui relèvent des mondes anciens. À côté de la Grèce et de Rome, figurent en effet, selon les versions du jeu, l'Égypte, les Perses, les Assyriens, Carthage, les Hittites, Babylone avec leurs dirigeants aux noms familiers: Ramsès II (fig. 17), Darius I $^{\text {er }}$, Assurbanipal, Didon, Nabuchodonosor II. Outre les traits qui sont liés à la "personnalité » de son dirigeant, chaque civilisation bénéficie d'atouts particuliers qui lui assurent une force par rapport à ses adversaires ${ }^{56}$. L'examen de ces capacités spécifiques permet de voir quels stéréotypes sont activés par le jeu. Ainsi, tandis que l'Égypte peut avancer rapidement dans l'édification des Merveilles grâce à son talent de "bâtisseur ", la Perse dispose d'un monument spécifique, la cour du Satrape, qui donne de la prospérité à sa population. La Grèce peut, elle, compter sur «la Ligue hellénique » qui lui procure de bonnes relations avec ses alliés. Parmi ses unités militaires spécifiques, elle dispose des hoplites et de compagnons de cavalerie (Companion Cavalry) qui remplacent les lanciers et les simples cavaliers. À Rome, ce sont les légions qui se substituent aux unités équivalentes. Le joueur doit savoir exploiter les forces de ses combattants, sans négliger ceux de l'adversaire. Tout l'intérêt du jeu est dans l'équilibre des forces. Le principe sous-jacent est finalement assez comparable à ce qui se joue dans les combats de gladiateurs romains, reposant sur des paires dissymétriques. Certaines unités sont très efficaces, mais coûteuses; d'autres seront bonnes pour l'attaque, mais plus mauvaises pour la défense. Les forces et les faiblesses de chacune doivent se compenser pour rendre le combat équilibré et l'issue incertaine.

\section{La Grèce et Rome, deux civilisations comme les autres ?}


Fig. 18 : Gorgô, reine de Sparte dans Civilization VI.

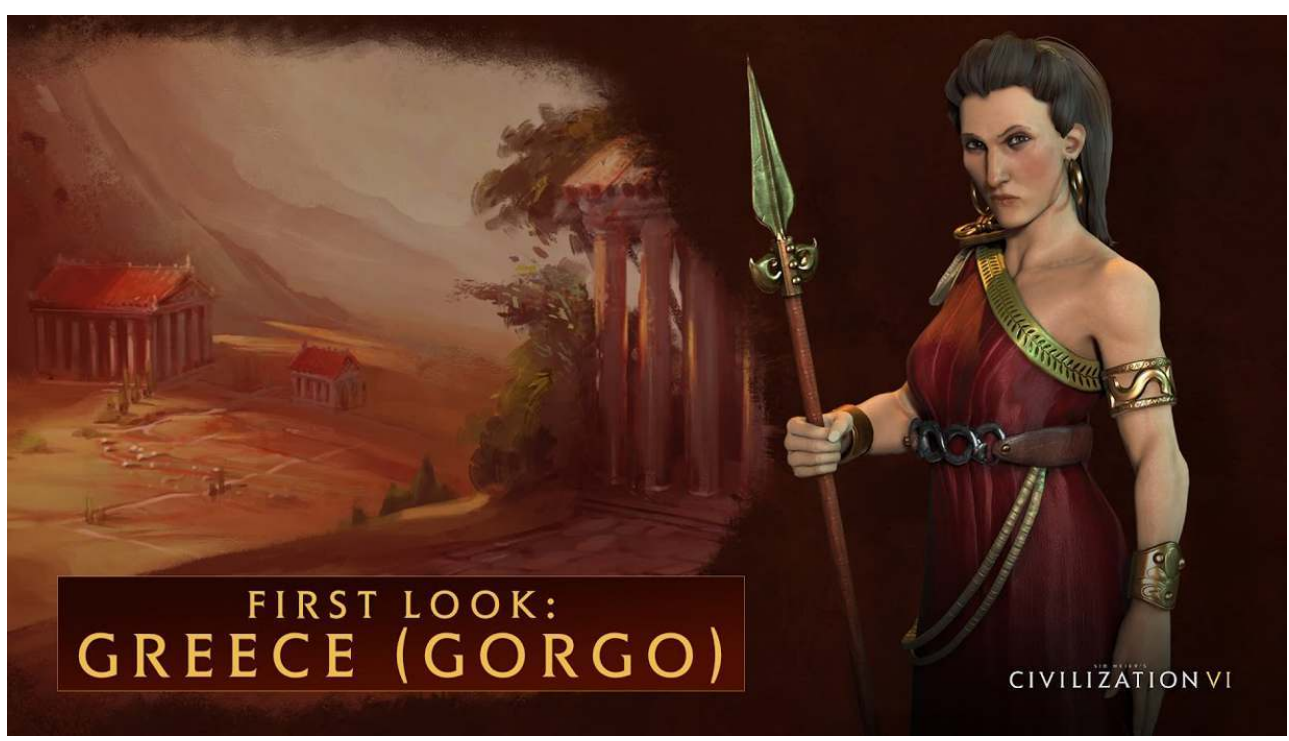

Capture d'écran @2K Games | Firaxis

La Grèce et Rome ne sont cependant pas exactement mises sur le même plan que les autres civilisations anciennes. Car, si, pour surprendre le joueur et lui donner de nouvelles possibilités, la liste des civilisations change d'une édition à l'autre ${ }^{57}$, on constate que la Grèce et Rome sont présentes dans toutes les versions du jeu de 1991 à 2016. Pour introduire un peu de variété, le dernier opus de la série a mis, à la tête de chacune de ses civilisations-vedettes, de nouveaux dirigeants: à Rome, Trajan a remplacé Octavien/ Auguste et pour personnifier la Grèce, le jeu offre désormais, à la place d'Alexandre ${ }^{58}$, un choix entre deux options, Périclès, l'Athénien ou Gorgô, reine de Sparte (fig. 18). Ces changements apportent de nouveaux « traits » de caractère à chacune de ces civilisations, ce qui permet au joueur d'expérimenter de nouvelles stratégies. Alors qu'Auguste incarnait la pax romana ${ }^{59}$, apportant des bonus à chaque nouvelle construction, Trajan, associé à la célèbre colonne, est d'abord un chef militaire. De même, Périclès sera un dirigeant exemplaire pour engager la Grèce dans une bataille culturelle, tandis que le tempérament plus agressif de la reine de Sparte sera plus indiqué pour mener des combats.

Pourtant, malgré les variations introduites (qui changent un peu le scénario, l'interface graphique et sonore et surtout la façon de jouer, le gameplay), Rome reste Rome, la Grèce demeure la Grèce. Cette permanence traduit une forme d'essentialisation des civilisations grecque et romaine, révélatrice du statut d'exception de ces deux cultures dans l'imaginaire occidental. Les spécialistes des jeux vidéo savent en effet combien ceux-ci sont dépendants des « goûts » du public : Rome et la Grèce ont un pouvoir d'attraction qui les rend probablement incontournables aux yeux des consommateurs ${ }^{60}$. Mais le jeu contribue aussi à entretenir ce statut privilégié en réaffirmant avec force la grandeur des deux civilisations et en soulignant la continuité historique qui les rattache à nous. Il suffit, pour s'en convaincre, de lire le texte qui présente la « Civilisation Grèce » au joueur et qui a pour fonction d'orienter son choix.

Il est difficile de surestimer l'impact que la Grèce a eu sur la culture occidentale et sur son histoire. La Grèce Classique a donné naissance aux plus grands artistes, philosophes, scientifiques, historiens, dramaturges et guerriers que le monde a 
jamais connus. Les soldats grecs et les colons ont répandu leur culture à travers la Méditerranée et jusque dans le Proche et le Moyen Orient. Les héritiers de la Grèce, les Romains, ont ensuite diffusé la pensée grecque en Europe et, à partir de là, celleci s'est répandue par-delà les océans et dans le Nouveau Monde. On doit à la Grèce et à son peuple un nombre étonnant d'inventions et de découvertes, dont la première performance théâtrale, l'histoire comme discipline, et le traité philosophique. Les Grecs ont fourni au monde occidental le premier événement sportif enregistré, la poésie, et le bâtiment dédié au théâtre. En politique, les Grecs ont créé la première démocratie connue et la république. L'influence grecque continue de se faire sentir, partout autour de nous: les médecins d'aujourd'hui prêtent toujours le serment d'Hippocrate et les architectes modernes tirent toujours leur inspiration des formes du classicisme grec. Dans une large mesure, la civilisation occidentale est la civilisation de la Grèce classique ${ }^{61}$. « nous » est également exprimé dans la Civilopedia, un outil en ligne entièrement dédié au jeu. Ainsi dans la section consacrée à « Rome », après avoir résumé les principales étapes de l'histoire romaine, depuis sa fondation jusqu'à la naissance de l'Empire, dans un style sérieux et didactique, cette encyclopédie ludique fait la liste de toutes les inventions héritées des Romains. Se déroule alors une sorte d'inventaire à la Prévert, dont la fantaisie hétéroclite est pour le moins déroutante : « les aqueducs, les vannes de bronze et les pompes à eau, le ciment à prise rapide et le béton, les mèches à feu, les parapluies, les loupes, les ciseaux, des formes de chaussures différentes pour le pied gauche et pour le pied droit, le calendrier moderne de douze mois $^{62} »$. Le joueur prend-il pour argent comptant ces informations? A t-il envie d'en savoir plus ${ }^{63}$ ? Les lit-il, même, avant de jouer? On peut légitimement en douter ${ }^{64}$. Mais ces textes, qui « accompagnent » le jeu et font partie de son environnement, n'en sont pas moins révélateurs de l'idéologie qui le sous-tend. présente cette civilisation souligne en effet l'extension géographique de l'empire et son caractère durable :

L'Empire romain est l'entité politique la plus remarquable et la plus durable de l'histoire de la civilisation Occidentale. Il fut fondé autour du vIII siècle avant J.-C. et il en subsista des portions jusqu'au XIV siècle de notre ère [...] Les Romains étaient un peuple de guerriers. Au sommet de leur puissance, ils régnaient sur un empire qui couvrait une large part de l'Angleterre, toute l'Europe occidentale, l'Afrique du Nord, l'Égypte, la Grèce et le Proche et Moyen Orient ${ }^{65}$ ». C'est donc cette longue présence dans un vaste espace qui leur permit de «modeler en profondeur la culture occidentale, dans le domaine des lois, de l'art, de l'architecture, de la religion, de la langue et de la guerre ${ }^{66}$.

Le rôle central joué par Rome comme modèle de puissance impérialiste se lit d'ailleurs aussi à travers la description des autres civilisations. À propos de l'Arabie par exemple, la notice précise: "Durant ses 600 ans d'existence, le califat connaitra une immense 
extension en taille et en puissance, dominant l'Espagne, le Nord de l'Afrique, le Moyen Orient, l'Anatolie, les Balkans et la Perse, gouvernant un Empire qui rivalisa au moins avec celui des Romains au faîte de leur puissance ». De même l'Empire Ottoman, né en Anatolie au XIII ${ }^{\mathrm{e}}$ siècle, «qui s'est étendu sur trois continents et qui a prospéré pendant six siècles ", «bien que relativement peu connu en Occident, [...] mérite une place de choix à côté de l'Arabie, de la Grande Bretagne et même de Rome ». Pour le joueur occidental contemporain, il est manifestement difficile de comprendre l'impérialisme en dehors de la référence à Rome, modèle inégalé67.

\section{Cités-États, Âge d'or et Merveilles : éléments topiques}

36 On peut cependant se placer à un autre niveau pour saisir la manière dont le jeu Civilization manie les références à l'Antiquité. En effet, un certain nombre de notions, d'éléments topiques, sont tirés de leur contexte et introduits dans le jeu pour construire un univers cohérent.

Certains de ces éléments sont implicitement liés aux réalités politiques des cités grecques. Le joueur se trouve ainsi amené à rencontrer des Cités-États (City-States). « Les cités-États représentent les civilisations mineures éparpillées aux quatre coins du monde. Elles se définissent par leur nom même: des États centrés sur une ville unique ${ }^{68}$ ». Elles ne peuvent pas gagner et ne constituent donc pas des adversaires, mais elles peuvent faciliter ou entraver le progrès vers la victoire. Il est donc préférable de les avoir pour alliées. On en trouve de différents types: certaines cités sont centrées sur la culture; d'autres sont des cités maritimes, d'autres encore sont des cités militaires ${ }^{69}$. Le nom de certaines de ces cités - Tyr, Ur, Byblos, Sidon - montre que le lien à l'univers de référence n'a pas complètement disparu. En même temps, on trouve aussi dans la liste de ces citésÉtats la plupart des capitales mondiales - de Buenos Aires à Kaboul, de Vancouver à Singapour en passant par Zurich ou Mogadiscio - ainsi que des villes qui ont joué un rôle central dans l'histoire du commerce international: Samarcande, Riga, Manille, Gênes, Panama. Ce mélange montre bien que la référence antique sert uniquement de matrice pour fabriquer une catégorie dont on a besoin dans l'économie du jeu. La cité-État a une utilité purement fonctionnelle.

Un autre exemple de la façon dont la référence à l'Antiquité «travaille l'imaginaire politique » de Civilization est l'usage des « doctrines sociales (social policies) ». Le joueur doit en effet choisir dans une liste un "mode de gouvernement» (la tradition, la piété, l'honneur, la liberté, l'ordre) et au cours du jeu, expérimenter toutes les «branches » de cette «doctrine sociale $»^{70}$. Ces doctrines ont des effets dans le jeu: certaines vont augmenter la production des cités, d'autres créer des richesses, tandis que d'autres encore seront plus efficaces pour créer une armée performante. Fait remarquable, aucune de ces « doctrines » n'est explicitement liée à l'Antiquité, puisque même la " démocratie » est affranchie de la référence athénienne: elle n'est pas disponible avant l'Ère de la Renaissance. 
Fig. 19 : Le tableau des « doctrines sociales » (Civilization V).

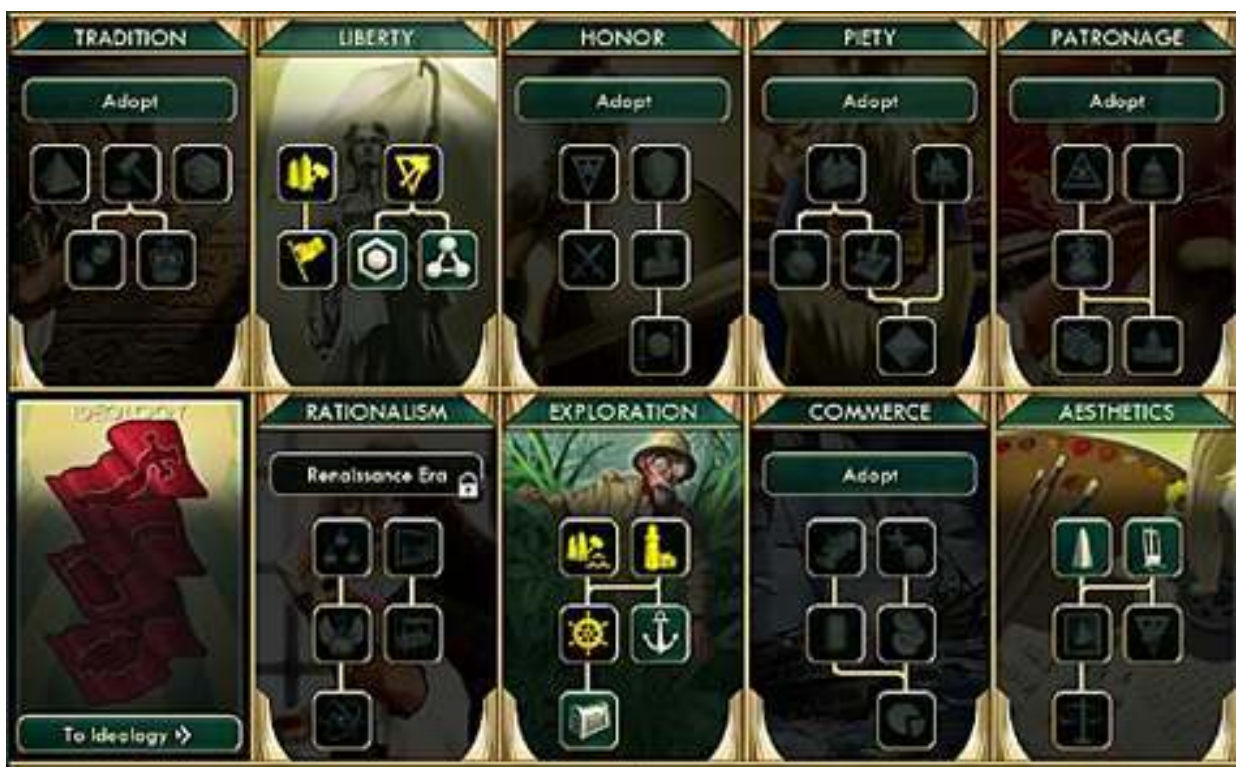

Capture d'écran @2K Games | Firaxis

39 Quant aux deux formes de liberté proposées par le jeu (Freedom et Liberty), elles ne peuvent se comprendre que dans le cadre de la culture américaine ${ }^{71}$. En revanche la présentation, sous forme de tableau, de ces différents «types de gouvernement», qui offrent chacun une série de "variantes » (fig. 19), l'espèce de relation généalogique qui lie ces doctrines entre elles ou qui les oppose les unes aux autres, n'est pas sans rappeler les « constitutions » d'Aristote ${ }^{72}$.

40 Si l'on quitte le domaine politique, deux autres éléments ${ }^{73} \mathrm{du}$ jeu font explicitement référence à la culture antique : l'Âge d'Or et les Merveilles. L'Âge d'Or (Golden Ages) est un élément lié au Bonheur de la population et aux Grands Hommes. L'acquisition d'un Artiste ou d'un Savant illustre permet en effet de créer pour sa civilisation une période de grande prospérité : « une période durant laquelle tout semble fonctionner parfaitement, comme si, soudain, les dieux ${ }^{74}$ nous avaient souri $»^{75}$. Cette période « bénie » dure plusieurs tours et permet d'accélérer le développement ou d'enrichir rapidement une civilisation, car chaque production bénéficie alors d'un rendement accru, les villes se remplissent d'or, la culture augmente ${ }^{76}$. 
Fig. 20a : L'une des Merveilles de Civilization V : le Colosse.

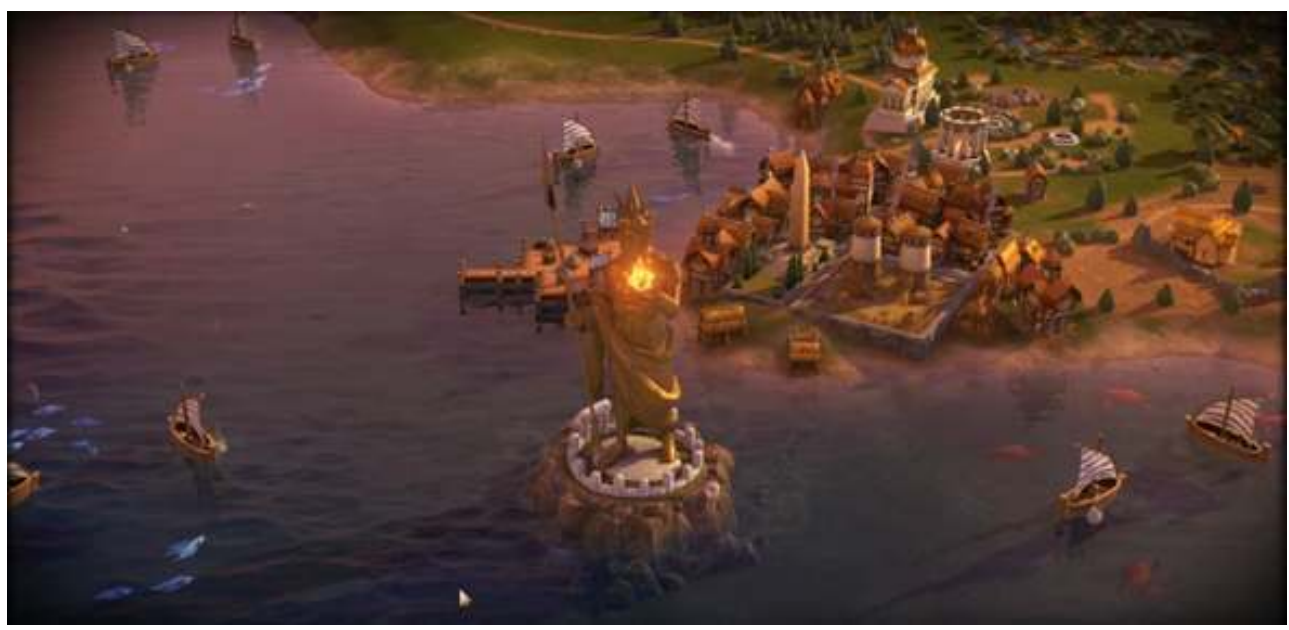

Capture d'écran @2K Games | Firaxis

Fig. 20b : Le Mausolée d'Halicarnasse (Civilization V).

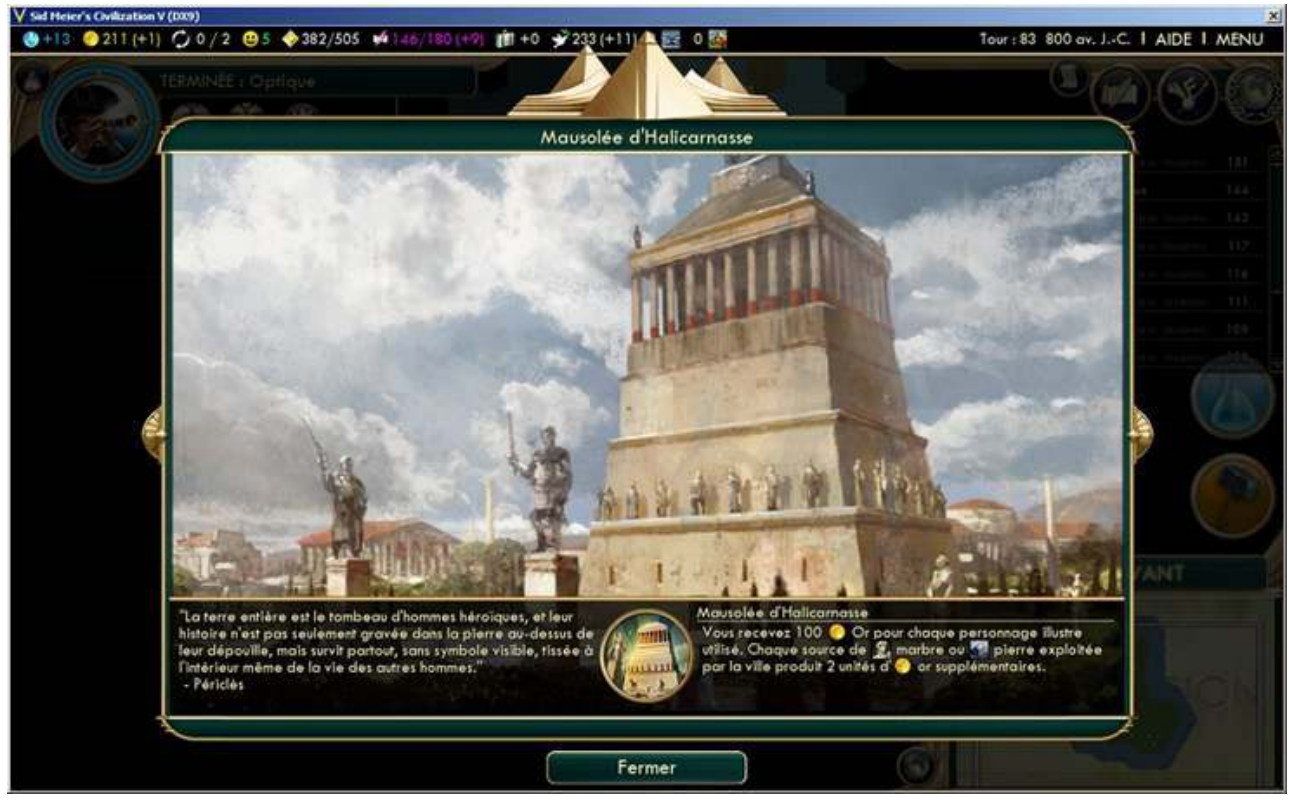

Capture d'écran @2K Games | Firaxis 
Fig. 20c : L'Oracle (Civilization VI).

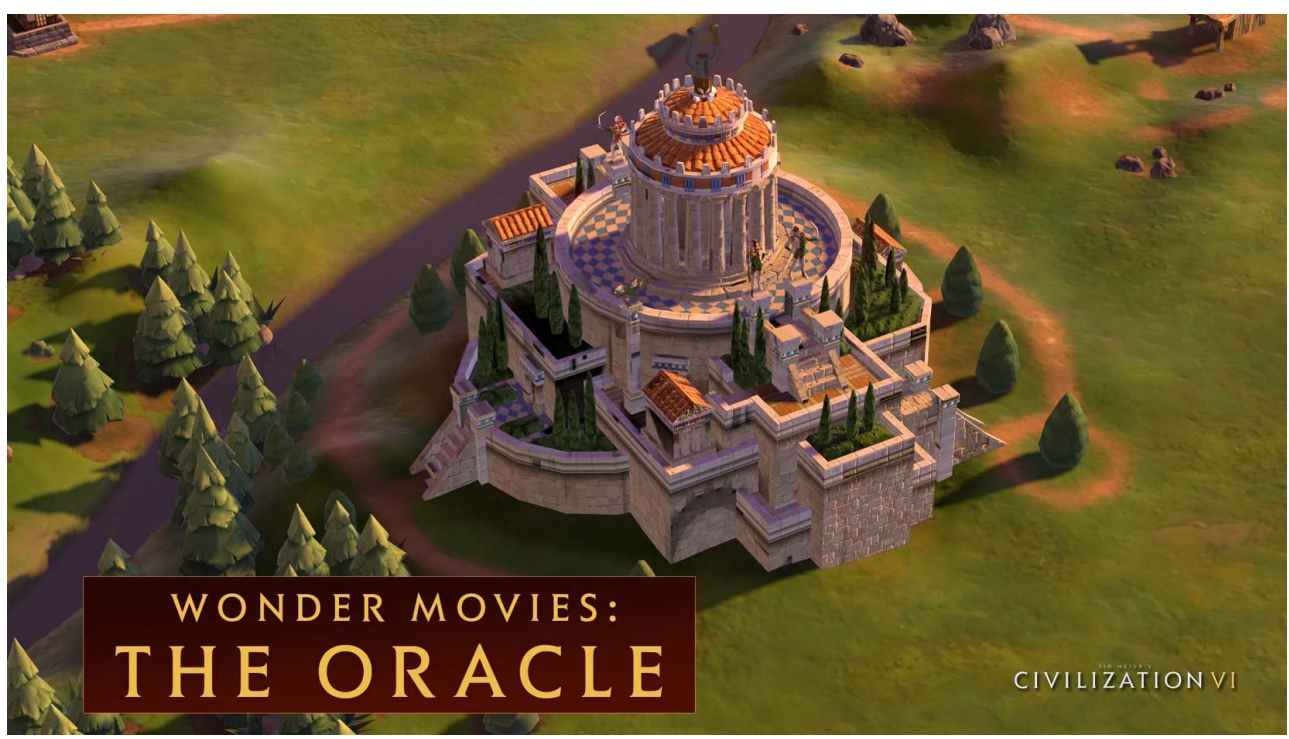

Capture d'écran @2K Games | Firaxis

Fig. 20d : La Grande bibliothèque (Civilization IV).

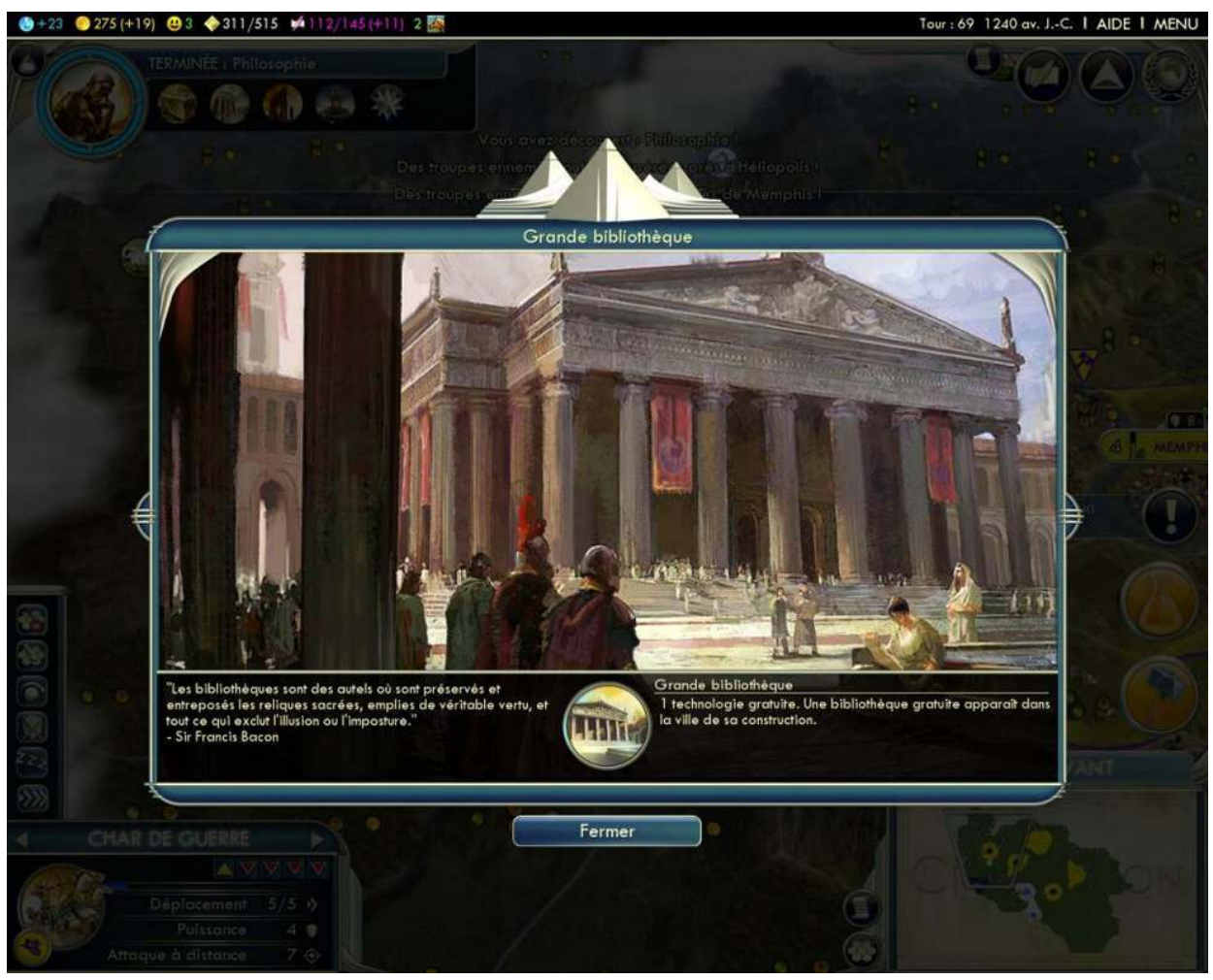

Capture d'écran (C2K Games | Firaxis

41 Plus intéressante encore, pour comprendre le rôle joué par l'imaginaire antique, est la place attribuée aux « Merveilles ». En effet, chaque civilisation est amenée au cours du jeu à découvrir et à protéger les Merveilles naturelles ${ }^{77}$ qui sont sur son territoire et à construire des monuments uniques, enviés de tous ${ }^{78}$. Ces Merveilles monumentales sont de deux types : mondiales, elles n'existent qu'en un seul exemplaire dans tout le jeu, elles 
sont très puissantes et très chères ; nationales, il peut y en avoir une seule de même sorte dans chaque civilisation ${ }^{79}$. La présence des Merveilles apporte des points de culture et du bonheur (Happiness) aux habitants d'une civilisation. Comment ne pas reconnaitre dans cet élément de jeu cette idée, héritée d'Hérodote, qu'une civilisation se signale par des sites et des monuments exceptionnels, les thaumata, dignes d'admiration et objets de description pour l'historien-enquêteur ou le périégète ${ }^{80}$ ? En outre, les Merveilles (Marvels ) du jeu ont un nom et, parmi elles, se trouvent toutes celles qui figurent dans la liste canonique des Sept Merveilles du monde et qui, de ce fait, appartiennent au monde antique : les pyramides, les Jardins suspendus, le grand phare, le temple d'Artémis, la grande bibliothèque, le colosse etc. Malgré leur « délocalisation » (on « oublie », dans leur dénomination, Gizeh, Babylone, Éphèse, Rhodes ou Alexandrie), ces Merveilles restent facilement identifiables (ne serait-ce que par leur graphisme) (fig. 20a, 20b, 20c et 20d) ; elles peuvent cependant, dans le jeu, être construites dans n'importe quelle cité de n'importe quelle civilisation : il suffit d'être le premier à avoir les ressources nécessaires. Autrement dit, la référence explicite au monde grec permet de faire fonctionner la Merveille en créant un effet immédiat de reconnaissance, mais la disjonction entre ces Merveilles et leur localisation "réelle » crée une tension inhérente à l'univers vidéoludique. En outre, dans Civilization, les Merveilles du monde antique, pour la plupart disparues, coexistent avec d'autres monuments célèbres, comme la Tour Eiffel, Sainte Sophie, l'Alhambra, l'Opéra de Sidney, la Tour de Porcelaine, Christ Rédempteur, La Grande Muraille, Stonehenge, etc. Tous ces éléments fonctionnent, de la même manière, comme des signifiants détachés de leur univers de référence ${ }^{81}$.

Fig. 21 : Un univers fictif à « effet Babel ».

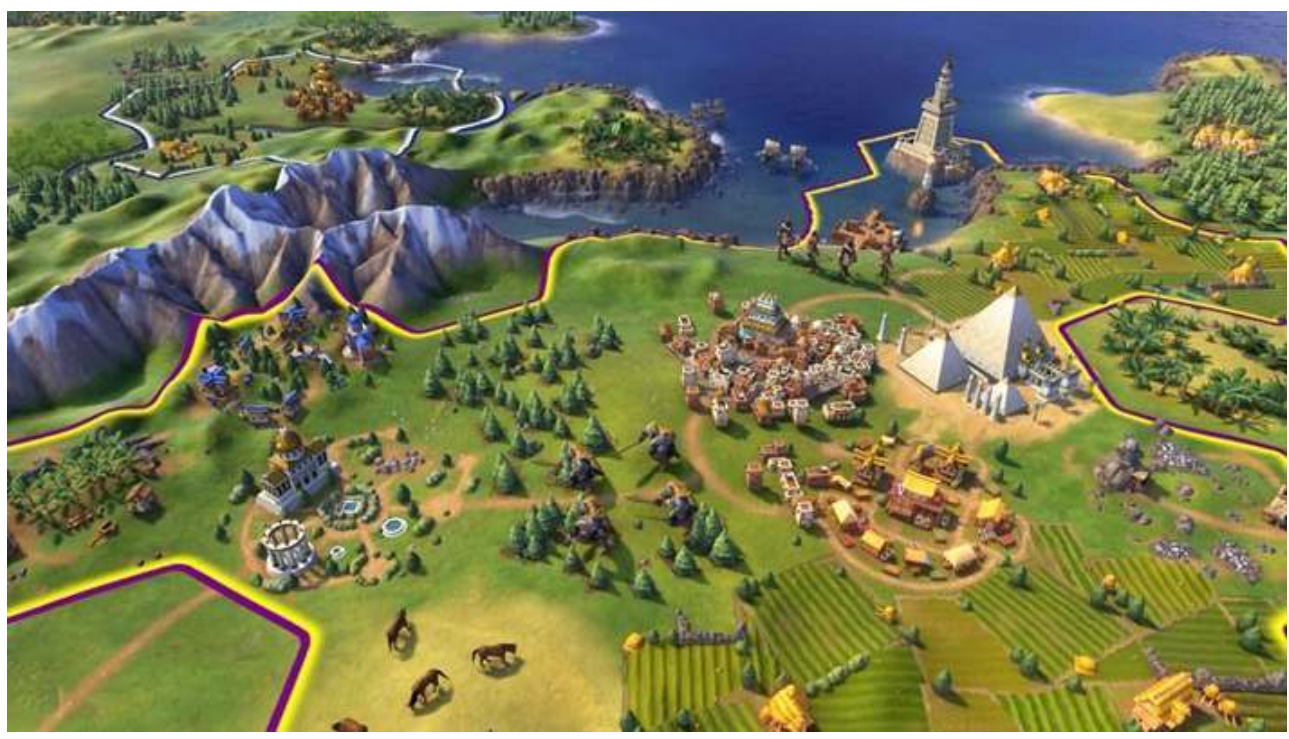

Capture d'écran @2K Games | Firaxis

Peut-on dire pour autant qu'elles sont une invention du jeu ? Non, car on a, sur le modèle antique, établi bien d'autres listes de Merveilles qui visent à inscrire au patrimoine de l'humanité les monuments et les sites les plus remarquables du monde contemporain ${ }^{82}$. La juxtaposition de ces différentes listes permet de voir que Civilization superpose, plus qu'il n'invente, divers ordres de réalité, différentes «mythologies ${ }^{83}$ " anciennes et contemporaines, et qu'il "joue» avec l'imaginaire des civilisations pour fabriquer un monde qui se présente comme une totalité (fig. 21). Un monde qui se laisse appréhender 
sur le mode de la "coprésence » et du "feuilletage » fait d'éléments hétérogènes et produisant ce qu'on a appelé un « effet Babel ${ }^{84}$.

Un dernier moyen d'utiliser la « référence antique » est le recours aux langues anciennes. En effet, la série Civilization fait un usage discret, mais efficace, des mots latins et on peut questionner la fonction de cet usage linguistique. Ainsi l'un des moyens de remporter la victoire est de battre ses concurrents sur le terrain technologique. Pour cela le joueur doit, avant les autres, acquérir dans l'ordre les diverses technologies qui sont disponibles au cours de sa progression, puis fabriquer les pièces nécessaires à la construction d'un vaisseau spatial pour envoyer des colons vers Alpha Centauri ${ }^{85}$. Ce nom, une fois encore, n'est pas une invention : il renvoie à un ensemble d'étoiles bien connu des astronomes, un système stellaire assez proche du système solaire. Mais son usage dans le contexte du jeu et surtout son association avec d'autres mots à consonance gréco-latine, comme Utopia ${ }^{86}$ ou encore le programme Apollo ${ }^{87}$, ont pour effet de détacher ce terme de son référent extra-ludique et de forger une langue spécifique, celle des jeux vidéo, un idiome partagé créant un univers de fiction. Cet usage du latin est évidemment très proche de celui qu'on trouve dans les romans et les films de science-fiction ${ }^{88}$.

Ce pouvoir de « fictionnalisation " prêté aux langues anciennes est aussi observable dans l'utilisation (ou plutôt le détournement) de citations. La civilisation Allemagne est ainsi définie par une "capacité spéciale " désignée par l'expression "furor teutonicus " ${ }^{89}$. Or cette expression qui permet de créer un trait spécifique, et même ethnique, est un emprunt littéraire : on la trouve dans un vers de Lucain pour désigner la fureur guerrière, proverbiale, du peuple des Teutons ${ }^{90}$. À quoi sert, dans ce cas, le recours au latin ? Il est probable que le latin permette de dire ce qu'aujourd'hui l'on ne pourrait décemment traduire, pas plus en anglais qu'en français. On peut donc se demander si, dans ce type de jeux, l'emploi des langues anciennes ne permet pas le passage du "sérieux" au "ludique ", s'il n'a pas le pouvoir de "déréaliser », et donc de vider les mots de leurs référents, et en particulier de leurs référents historiques. Ou au contraire, dans le cas de la « fureur teutonne », s'il ne permet pas de les réactiver subrepticement.

\section{Conclusion}

Il s'agissait, on l'aura compris, de cerner quelques-uns des enjeux idéologiques d'un jeu vidéo dont le succès planétaire et exceptionnellement durable ${ }^{91}$ permet de penser qu'il exerce un rôle non négligeable dans la transmission des stéréotypes liés à la conception occidentale de la - et des - civilisation(s) : les critères qui les définissent, les processus qui les font et les défont, les différentes façons d'asseoir leur pouvoir sur le monde. Il montre enfin et surtout la manière dont l'Occident contemporain se définit perpétuellement dans un rapport de continuité et de filiation directe avec l'Antiquité gréco-romaine.

En rappelant ces stéréotypes, on pourrait s'étonner du décalage existant entre l'attachement au passé manifesté par les créateurs du jeu - l'omniprésence de l'Histoire dans les scénarios complexes qu'ils inventent et réinventent ${ }^{92}$ - et l'absence de volonté de faire connaître ce passé ou cette histoire, au-delà des clichés. Cette question de l'historicité des contenus de Civilization rejoint d'ailleurs celle de l'usage qui peut être fait de tels jeux dans les pratiques pédagogiques ${ }^{93}$. En effet, comme le cinéma ou la bande dessinée, les jeux vidéo dits « historiques » intéressent l'école. Certains enseignants, au Québec notamment, y ont réfléchi et s'y sont essayé, parfois avec succès ${ }^{94}$. 
Cependant, ce regard et cet usage purement didactiques du jeu vidéo tendent à oblitérer ce qui en fait la saveur et le succès, sa capacité à créer un monde, ou des mondes, à la fois proches et lointains, où se mêlent le passé le plus ancien et les préoccupations du présent le plus actuel, la vraisemblance historique et la fantaisie la plus totale. Or les références à la Grèce et à Rome permettent ces allers et retours permanents.

Car, si l'Antiquité est conçue dans ce jeu comme le temps des origines et le lieu où se sont déployées d'importantes civilisations, dont nous serions culturellement les héritiers, elle apparaît aussi comme un lieu de déréalisation, un espace où la frontière entre la réalité et l'imaginaire, entre l'histoire et le mythe, est suffisamment poreuse pour favoriser la création d'univers vidéoludiques. L'Antiquité favorise les voyages dans le temps et dans l'espace, permet l'uchronie et la mise à distance, tout en immergeant le joueur occidental dans un univers qui lui est familier. Et c'est probablement l'une des raisons de son succès et de sa fascinante « résistance ».

\section{BIBLIOGRAPHIE}

André L.-N. et Lécole-Solnychkine S. (2011), « La référence à l'antique dans les jeux vidéo : paysages et structures mythologiques ", dans Rufat S. et Ter Minassian H. dir., Les jeux vidéo comme objet de recherche. Questions théoriques, p. 88-102.

André L.-N. et Lécole-Solnychkine S. (2013), « L'Antiquité vidéoludique, une résurrection virtuelle? », Nouvelle Revue d'Esthétique 11, p. 87-98.

Aziza C. (2011), « L'Antiquité dans la science-fiction : une histoire fantasmatique », Anabases (en ligne) 13, consulté le 21 février 2018.URL : http://journals.openedition.org/anabases/1900 ; DOI : $10.4000 /$ anabases. 1900

Barthes R. (1957), Mythologies, Paris.

Ben Achour Y. (2007), « Les relations entre les civilisations islamique et occidentale » Jura Gentium (Rivista di Filosofia del Diritto internazionale e della Politica globale), IV, War, Law and Global Order, p. 107-122 ; consultable en ligne : http://www.juragentium.org/Centro_Jura_Gentium/ la_Rivista_files/JG_2007.pdf

Boutonnet V., Joly-Lavoie A. et Yelle F. (2014), « Intégration des jeux vidéo : entre jeux sérieux et jeux traditionnels ", dans M.-A. Éthier, D. Lefrançois et S. Demers dir., Faire aimer et apprendre l'histoire et la géographie au primaire et au secondaire, Sainte-Foy, Multimondes, 2014, p. 303-322.

Corbeil P. et Laveault D. (2013), « Validity of a Simulation Game as a Method for History Teaching », Simulation Gaming 42-4, p. 462-475.

Dalongeville A. (2001), L'image du barbare dans l'enseignement de l'histoire : une expérience de l'altérité, Paris.

Dumézil B. dir. (2016), Les Barbares, Paris.

Gérard A. (1998), « Le grand homme et la conception de l'histoire au XIX siècle », Romantisme, 100 : Le Grand Homme, p. 31-48. 
Huntington S. (1996), The Clash of Civilizations and the Remaking of World Order, Paris ; trad. fr. Le choc des civilisations, Paris, 1997.

Huizinga J. (1988), Homo ludens. Essai sur la fonction sociale du jeu, Paris (1 ${ }^{\text {ère }}$ éd. néérl. 1938).

Joly-Lavoie A. et Yelle F. (2016), « Le jeu vidéo pour enseigner l'histoire : synthèse d'une approche théorique et pratique ", TRACES 54-3, p. 19-24; consultable en ligne : https ://ledidacticien. com/2016/06/27/le-jeu-video-pour-enseigner-lhistoire-synthese-dune-approche-theorique-etpratique/

Rabino T. (2013), « Jeux vidéo et histoire », Le Débat 5-177, p. 110-116.

Rolling A. et Morris D. (2000), Game Architecture and Design, Scottsdale.

Ter Minassian H., Rufat S. et Coavoux S. dir. (2012), Espaces et temps des jeux vidéo, Paris.

\section{NOTES}

1. Laury-Nuria André et Sophie Lécole-Solnychkine (2013) relèvent ce paradoxe au début de leur article.

2. J'utilise cette expression en référence au titre d'un récent colloque, Antiquipop, organisé par Fabien Bièvre-Perrin (IRAA), Élise Pampanay et Laury-Nuria André (HiSoMA), à l'Université Louis Lumière-Lyon II, du 26 au 28 mai 2016, consacré aux divers usages et manifestations de l'Antiquité dans la culture populaire contemporaine.

3. Parmi quelques-uns des jeux les plus connus, citons par exemple Ages of Empires I, Caesar IV, Gates of Troy, Spartiates, CivCity Rome, Rise of the Argonauts, Rome Total War I et II.

4. Ce jeu est sorti dans sa première version en 1991. Il est le premier et, à ce titre, il est devenu la référence d'un genre de jeux vidéo dits "de civilisation ", comme Age of Empires, Rise of Empires, etc.

5. Civilization VI est sorti aux États-Unis et en Europe en octobre 2016. Parmi les extensions, citons par exemple Civilization III : Conquests (2003) ; Civilization IV : Warlords (2007) ; Civilization IV : Beyond the Sword (2007) ; Civilization V: Gods and Kings (2012).

6. La partie est censée débuter en 4000 av. J.-C. et finir au plus tard en 2050 de notre ère.

7. On peut jouer en solo, contre l'ordinateur, ou en mode «multi-joueurs », via Internet. Et il est possible d'affronter entre une et six civilisations.

8. Le caractère évolutif du jeu et, en raison de son succès, la poursuite ininterrompue de son développement depuis sa sortie initiale, en a fait l'un des jeux les plus riches en termes de scénario, d'interface (graphisme, son, qualité des animations) et de possibilités de jeu. Chaque nouvelle version est unanimement saluée par la critique spécialisée.

9. Cette expression « se prendre pour » n'est évidemment pas exacte. La pratique ludique ne rend pas schizophrène. Dans la terminologie du jeu vidéo, le joueur, pendant la durée du jeu, est un « avatar » de Napoléon.

10. Uchronie est un néologisme inventé par Charles Renouvier en 1857, forgé sur le mot utopie. Dans son acception précise, ce mot renvoie à une fiction se déroulant dans un monde en tout point similaire au nôtre, jusqu'à un certain événement qui, lui, diffère de ce qui s'est réellement produit et qui ouvre la voie à un nouvel agencement des faits. On peut cependant prendre ce mot dans un sens plus large pour faire référence à une fiction 
organisée chronologiquement, multipliant les références historiques, mais recomposant ces éléments pour créer un autre monde, et une autre Histoire.

11. Le temps de jeu est divisé en périodes (mais ce sont des étapes de développement, plus que des époques historiques): Ancient Era, Classic Era, Medieval Era, Renaissance Era, Industrial Era, Modern Era, Atomic Era, Inform Era.

12. Le Choc des civilisations (en anglais: The Clash of Civilizations and the Remaking of World Order) est le titre d'un essai d'analyse politique rédigé par l'Américain Samuel Huntington, paru en 1996 (trad. française, Paris, 1997). Cet essai, qui a suscité de nombreux débats, montre la nécessité de créer un nouveau modèle conceptuel pour décrire le fonctionnement des relations internationales après l'effondrement du bloc soviétique. Il s'appuie sur une description du monde fondée non plus sur des clivages "politiques», mais sur des oppositions culturelles, dites "civilisationnelles », dans lesquelles la religion tient une place centrale. Après avoir décrit, dans une première partie, un monde multipolaire, divisé en huit "civilisations" (civilisations chinoise, japonaise, indienne, islamique, occidentale, latino-américaine, africaine et orthodoxe), il montre le caractère inéluctable des guerres inter-civilisationnelles et de l'effacement de l'Occident. Si la première version de Civilization est sortie quelques années avant la parution de l'ouvrage d'Huntington, le succès du jeu et, plus globalement, de tous les jeux vidéo dits de "civilisation » depuis plus de vingt ans, montre la vulgarisation et même l'intériorisation de ses théories. Par la place donnée à la religion dans son scénario, c'est la dernière version du jeu, Civilization VI, qui porte la marque la plus évidente de cette idéologie (voir infra, note 13).

13. "There are many paths to choose from " (Civilization V, guide in line, p. 3). Le joueur peut en effet gagner en remportant une victoire diplomatique, économique, technologique ou culturelle. Civilization s'est d'ailleurs toujours présenté comme un jeu non militariste. Ce "pacifisme" affiché est particulièrement visible dans Civilization IV (2006) dont tout l'argument de vente repose sur le propos humaniste véhiculé par le jeu et dont le scénario souligne l'importance de la culture et de l'écologie. Au point qu'un site web consacré aux jeux vidéo, Grospixels, a pu écrire : «[...] la meilleure façon de gagner est d'être une nation démocratique, laïque et écolo. [...] le joueur progressera bien plus vite si elle n'est jamais troublée par la guerre [...] aucune des conditions de victoire n'implique d'être le plus riche. Civilization IV, un vrai jeu de gauche?» (https:// www.grospixels.com/site/civilizationiv.php).

Plus symptomatique encore du lien entre l'évolution du jeu et l'actualité géopolitique, la version la plus récente, Civilization VI (2016), a, parmi ses options, conservé la « victoire culturelle » et la « victoire scientifique », mais supprimé la « victoire diplomatique » pour la remplacer par la « victoire religieuse » : pour gagner, le joueur doit fonder une religion, avoir un prophète, construire des temples, envoyer des missionnaires et surtout convertir les autres civilisations à sa propre religion.

14. «Pourrez-vous mener votre civilisation vers la victoire ou finirez-vous écrasé sous les roues du char de vos ennemis, pour venir grossir les rangs de tous les chefs oubliés des peuples vaincus? ? (trad. personnelle, Civ. V, guide in line, p. 3).

15. Civilization est un jeu, mais il n'est pas dénué de sérieux. On peut en effet discuter la pertinence de la distinction, en usage chez ceux qui étudient les jeux vidéo, entre les « serious games " à vocation éducative et les jeux de pur divertissement. Voir André et Lécole-Solnychkine 2013, p. 88 et note 4. La porosité entre les genres est particulièrement sensible avec des jeux comme Civilization dont l'univers ludique est tissé de références 
historiques et culturelles et surtout qui comporte, à l'intérieur même du jeu et dans les divers outils qui l'accompagnent, de petites synthèses destinées à "informer » les joueurs. Outre la notice de jeu, très longue, le joueur a à sa disposition une encyclopédie en ligne, la "Civilopedia », fournissant toutes sortes de fiches sur les personnages, les civilisations, les monuments rencontrés. Le propos n'est pas de discuter la qualité de ces notices, mais de montrer la volonté affichée des concepteurs du jeu de fournir du contenu didactique.

16. Les États-Nations sont devenus majoritaires dans les éditions récentes: les civilisations ont pour noms Espagne, Norvège, France, Allemagne, Angleterre, Brésil etc.

17. Y. Ben Achour (2007, p. 108) rappelle le caractère flou et très arbitraire de la notion de civilisation: sa définition dépend d'un observateur qui sélectionne un ou plusieurs critères pour englober sous une même dénomination des réalités très différentes. Tantôt prévaut le critère géographique (civilisation africaine, amérindienne), tantôt le critère linguistique (civilisation latine ou grecque); tantôt on retient un critère ethnique (civilisation celte) ou religieux (civilisation islamique). L'expression "civilisation occidentale » a perdu toute connotation géographique pour désigner, dans son sens actuel, «l'ensemble des cultures de souche européenne, juxtaposant à leur patrimoine classique les acquis de la modernité, c'est-à-dire la science et la technologie industrielle et numérique, la sécularisation de la vie sociale et politique, le mode de gouvernement libéral et démocratique. »

18. Dix-neuf volumes sont parus aux éditions Arthaud entre 1961 et 1983, tous rédigés par des spécialistes faisant autorité dans leur domaine: deux volumes sur l'Europe par P. Chaunu, l'Occident médiéval par J. Le Goff, la Renaissance par J. Delumeau, la Grèce archaïque, classique et hellénistique (2 vol.) par F. Chamoux, la civilisation romaine par $\mathrm{P}$. Grimal. On trouve aussi dans cette collection, nettement centrée sur l'histoire occidentale, des volumes consacrés à l'Égypte, au Japon, à la Chine classique, à l'Inde et à l'Islam classique.

19. Cette vaste collection comprend trente-cinq titres. Outre des "classiques " comme Sumer, la première des Civilisations, l'Égypte des Pharaons, la Civilisation étrusque, la Grèce du Parthénon, la Civilisation des Aztèques, des Incas, des Celtes, des Vikings, La Perse des Grands rois et de Zoroastre, on trouvait un livre consacré à l'Atlantide, un autre aux Civilisations méconnues de la Mer rouge ou encore aux Civilisations des steppes et à L'île de Pâques. La préhistoire n'était pas oubliée (La civilisation des mégalithes), pas plus que le christianisme ( La Civilisation chrétienne primitive).

20. Selon Sid Meyer, le créateur du jeu, c'est précisément l'essence même des jeux vidéo que d'offrir « des séries de choix intéressants » (cité dans Rolling et Morris 2000, p. 38).

21. Civilopedia, Civ. V, s.v. «Songhai ».

22. Un autre exemple de ce cynisme affiché est présent dans la description qui est faite de l'Or : «It may be true that 'money can't buy you love', but it can purchase a submarine armed with nuclear missiles, and that's not bad !» (Civ. V, guide in line, p. 103).

23. Un autre élément révélateur du statut spécifique des civilisations « exotiques » est leur interchangeabilité. En effet, pour garder à peu près toujours le même nombre d'options (autour de dix-huit) et pour offrir de nouvelles possibilités de jeu, chaque nouvelle version remplace quelques civilisations par quelques autres. Or, si les « grandes civilisations » et les États-Nations changent peu d'une édition à l'autre, les Zoulous ou le 
Mali disparaissent au profit des Songhaï, l'Indonésie se substitue à Siam ou aux Khmers, les Iroquois aux Shoshones etc.

24. Cette volonté de faire une histoire plus politiquement correcte du point de vue du genre apparait notamment dans le choix, fait par Civilization $V$, de mettre une femme à la tête de la Chine, l'impératrice Wu Zetian (625-705). «Like most civilizations, China has been male-dominated throughout much of its history. [...] For a woman to attain the rank of Emperor, to become the most powerful person in China was almot unheard of. Only one person in the entirety of Chinese history was able to do so: Wu Zetian» (Civ. V, s.v. "Wu Zetian»). Dans CIvilization VI, la Scythie est également dirigée par une femme, Tomyris.

25. Voir Civilization IV.

26. En réalité, cette évolution contre-historique est originellement due à un défaut dans l'intelligence artificielle du jeu. En effet si, dans les premières versions, la civilisation Inde était dotée d'attributs pacifiques, l'agressivité de Gandhi et son goût pour les armes nucléaires se développaient parfois au cours de la partie au point d'en faire un des dirigeants les plus dangereux de l'ère contemporaine. Les joueurs ont vite repéré ce bug et échangé des blagues en ligne (surnommant ce nouveau personnage Nuclear Gandhi). Devenu viral, ce phénomène a provoqué l'envie, chez les éditeurs du jeu, de modifier le scénario en conséquence et de proposer dans Civilization $V$, un Gandhi ultra militariste (fig. 10b).

27. Le joueur peut, en début de partie, choisir parmi différents espaces géographiques : une carte ressemblant à la terre faite de grands continents et de quelques îles; ou la pangée (un continent unique) ; ou la formation en archipels (aucun continent et beaucoup d'îles). Plus généralement, sur l'espace dans le jeu vidéo, voir le récent volume de Ter Minassian et al. 2012.

28. Comme l'expliquent très bien $\mathrm{S}$. Rufat et $\mathrm{H}$. Ter Minassian dans l'introduction du volume cité supra, dans le jeu vidéo, l'écoulement du temps est toujours un défi spatial et l'espace est la matérialisation, à l'écran, du temps de jeu.

29. La ville produit de la nourriture, des biens, de l'or, de la science, de la culture. Le joueur peut choisir de produire tous ces éléments de manière équilibrée ou au contraire de se focaliser sur la production d'un type d'éléments.

30. Voir André et Lécole-Solnychkine 2011 : «L'implantation des infrastructures urbaines selon le quadrillage de la plateforme de jeu réfère tout aussi bien à la centuriation romaine qu'à la grille jeffersonienne américaine et risque fort de générer, à terme, un plan d'urbanisation qui possède tous les traits du lotissement moderne. »

31. En cas d'accroissement démographique trop rapide, la règle du jeu recommande de procurer davantage de divertissement à la population (« demand more stuff to keep them amused », Civ. V, p. 107). L'édification de bâtiments comme le Colisée, le Grand Cirque, le Théâtre, etc., que l'on peut multiplier et placer n'importe où sur le territoire, apaise alors le mécontentement.

32. "A city that has no building is pretty weak and primitive and will probably remain fairly small, while a city with a lot of buildings can indeed grow to dominate the world » ( Civ. V, guide in line, p. 75).

33. Sur l'histoire de la barbarie et de l'opposition barbare/civilisé, voir le récent livre collectif dirigé par Bruno Dumézil (2016, introduction et p. 1-89). 
34. «Barbarians are roving bands of villains who hate civilization and everything that goes with it » (Civ. V, guide in line, p. 65).

35. Rufat et Ter Minassian 2012 : «Les actions violentes et belliqueuses sont souvent un passage obligé de la domination des espaces vidéoludiques, même lorsqu'elles ne sont pas centrales. »

36. La guerre est l'état permanent des barbares (Civ. V, guide in line, p. 54). Ce trait reprend un stéréotype ancien que l'on retrouve aussi bien dans la vision des Gaulois par César que dans celle des Germains par Tacite.

37. Dans une des extensions du jeu Civilization IV: Warlords, le scénario prévoit que l'on puisse jouer les barbares. Dans Civilization VI, les barbares semblent s'être inspirés du personnage et de la mythologie de Conan, tant ils sont redoutables.

38. Lorsqu'un nouveau camp barbare apparaît dans une zone déjà explorée, un bruitage caractéristique se fait entendre. Ce cri est un signal qui doit inciter le joueur à chercher où se cachent les barbares, pour les débusquer avant qu'ils n'attaquent.

39. "If the entire world is civilized, the barbarians will be gone " (Civ. V, guide in line, p. 66).

40. On peut aussi choisir la " victoire aux points » : dans ce cas, on arrête le jeu en 2050 et on déclare vainqueur celui qui à cette date a accumulé le plus de points.

41. Le sous-titre de la série, qui lui sert de slogan, est « Build a civilization that will stand the test of time ».

42. La découverte de ruines antiques permet de disposer d'une "technologie gratuite", ou d'un terrain, d'un trésor (or), d'armes, de points de culture ou même de survivants qui viennent grossir la population!

43. «Ancient Ruins are the remnants of even earlier civilizations which rose and fell long before you came on the scene » (Civ. V, guide in line, p. 64).

44. Toutefois, dans Civilization, la ruine disparaît dès qu'on s'en est emparée. Autrement dit, il n'existe aucune esthétique des ruines ni même de valorisation de la ruine en tant que telle.

45. «It was advances in the technologies of agriculture and fishing that allowed cities to grow and thrive. It was advances in weaponry and masonry that allowed some cities to drive off the jealous barbarians who sought to steal their food and plunder their wealth. It was advances in medicine and sanitation that fought off the other great threat to civilization: disease. Advancing technology makes civilization stronger, bigger, smarter, and a much tougher opponent. It is critically important for a civilization to keep up technologically with its neighbors » (Civ. V, guide in line, p. 82).

46. Chaque apprentissage demande un certain nombre de tours et un tableau permet de voir ce que chaque technologie "débloque». Au début du jeu, seules quelques technologies sont disponibles : l'élevage, le tir à l'arc, la poterie, le travail de la mine. Les autres technologies s'appuient sur des technologies préalables. Par ailleurs, les technologies permettent d'obtenir des unités spécifiques (le tir à l'arc est par exemple nécessaire pour pouvoir disposer d'archers) ou de faire apparaitre des ressources stratégiques, non visibles au début du jeu : le travail des métaux permet d'acquérir le fer, l'élevage et le cheval. D'une certaine manière, on peut donc dire que la «culture » précède la « nature » dans le jeu. 
47. Plus qu'à des périodes historiques, ces «ères » correspondent à des degrés de civilisation.

48. La navigation, l'écriture et le calendrier nécessitent par exemple l'acquisition préalable de l'art de la poterie. La navigation est un préalable à l'invention de l'optique ; le travail du métal à celle de la physique ; etc.

49. Le rôle essentiel donné à l'agriculture est justifié par l'importance de la nourriture produite en abondance dans le développement des civilisations. "While humans had to spend virtually every waking moment hunting and gathering food for themselves and their families or tribes, they had little time or energy for other pursuits [...] Once surplus food is available, all else is possible» (Civ. V, guide in line, p. 77). Cette conception de la civilisation est très ancienne. On la trouve chez Condorcet dans son tableau de l'évolution humaine (Esquisse d'un tableau historique des progrès de l'esprit humain, 1794-1795): «la population s'accroît à mesure que les moyens de vivre deviennent moins périlleux et moins précaires ; l'agriculture, qui peut nourrir un plus grand nombre d'individus sur le même terrain, remplace les autres sources de subsistance : elle favorise cette multiplication, qui, réciproquement, en accélère les progrès » (Wikisource, p. 8). Cette idée concorde aussi avec la définition de la civilisation par l'archéologue Gordon Childe dans un ouvrage, Urban Civilization, paru en 1950. D'après lui, les premières civilisations sont Sumer, l'Égypte antique, la civilisation de la vallée de l'Indus et la civilisation chinoise. Les ensembles monumentaux qu'ont laissés ces civilisations les différencient des établissements du Néolithique. C'est, d'après lui la découverte, puis la maîtrise de l'agriculture qui ont ensuite entraîné une nouvelle organisation de l'espace et de l'activité humaine au sein de « civilisations urbaines ».

50. Dans Civilization V et VI, les prophètes font également partie des "personnages illustres ", signe de l'importance croissante donnée à la religion dans l'évolution du jeu. Indispensables à la fondation d'une religion, ils ne sont disponibles qu'au début du jeu (avant l'Ère de la Renaissance) et surtout ils sont en nombre limité. Dans la liste des prophètes figurent des personnalités aussi variées que Confucius, Lao Tseu, Jean le Baptiste, Irénée de Lyon, Siddartha Gautama ou Zoroastre. Moïse et Mahomet brillent par leur absence.

51. Dans les versions les plus récentes du jeu, la liste des Grands Personnages s'enrichit cependant de noms beaucoup moins attendus: on trouve par exemple, parmi les musiciens, K.P.H. Notoprojo, célèbre joueur de gamelan, un instrument javanais, ou parmi les savants, Naburimannuun, astronome chaldéen. Les listes sont très longues et la lecture de ces inventaires disparates, disponibles en ligne, fait également partie du plaisir du jeu.

52. Cette possibilité, laissée au joueur, de créer des personnages, de leur donner des noms, d'influer sur le scénario, lui donne un statut de co-producteur, caractéristique de l'univers vidéo-ludique, qui dépasse la question rebattue de « l'interaction ».

53. En témoigne par exemple l'édification du Panthéon, commencé par Soufflot et achevé, à l'initiative de l'Assemblée constituante, en avril 1791, par Quatremère de Quincy, monument laïc à la mémoire des grands hommes. Le rôle des Grands hommes dans la marche évolutive de l'histoire est également souligné dans l'ouvrage de Condorcet (cité supra, note 49 ; par ex. dans la « Huitième époque »).

54. Voir Gérard 1998. 
55. Même si la notice précise que le jeu commence vers 3500 av. J.-C. et si une sorte de compteur permet au joueur de suivre le passage du temps, les ères sont d'abord conçues comme des étapes de jeu.

56. «Mastering a civilization's strengths - and exploiting your enemies' weaknesses - is one of the most challenging parts of the game » (Civilization V guide in line, p. 18).

57. Voir supra note 26.

58. Alexandre était présenté dans la notice de Civilization $V$ comme l'un des plus grands chefs militaires de tous les temps, qui réussit «en dix-sept ans à peine à conduire son armée de victoire en victoire en Europe, en Asie, en Afrique et au Moyen Orient. Ces conquêtes lui ont permis de diffuser la culture grecque dans tout le monde connu et d'imposer le grec comme langue de culture pendant des siècles » (p. 180).

59. Dans Civilization V, Auguste est décrit comme « le premier et peut-être le plus grand empereur romain ", qui mit fin aux guerres civiles et fut à l'origine d'une période de paix qui dura deux cents ans. Il est aussi associé à l'âge d'or de la culture et de la littérature romaines. Le trait qui lui est associé est « la Gloire de Rome».

60. Ce pouvoir d'attraction se mesure aussi à travers la multiplicité des jeux qui ont pour support l'Antiquité gréco-romaine. Voir les exemples cités dans André et LécoleSolnychkine 2011 ; supra note 3.

61. Civ. V, guide in line, p. 180 (traduction personnelle).

62. Civilopedia, Civ. V, s.v. Rome (fin de notice)

63. Le jeu suscite de nombreux échanges entre les internautes, qui non seulement discutent en ligne des stratégies de jeu et échangent des tuyaux, mais encore s'interrogent sur les informations historiques qu'ils ont glanées dans l'environnement du jeu.

64. Il faudrait faire une enquête auprès des joueurs pour comprendre exactement comment se fait la sélection d'une civilisation, l'adoption d'une stratégie etc., mais il semble, au vu des questions posées à quelques joueurs de ma connaissance, que le choix d'une civilisation se fasse essentiellement sur des critères internes au jeu (en fonction des caractéristiques attachées à telle ou telle, des stratégies possibles au vu de ces caractéristiques), et non en fonction de l'identité ou de l'histoire réelle de cette civilisation. Cependant le prestige de Rome semble assez partagé.

65. Civ. V, Guide in line, p. 186 (traduction personnelle).

66. Ibid.

67. Ceci est d'ailleurs affirmé clairement à la fin de la notice sur Rome dans la Civilopedia : «Le puissant Empire romain est une entité complexe qui ne cesse de fasciner le monde contemporain. Bien qu'il ait disparu depuis longtemps, son ombre plane toujours sur le globe et les événements dont Rome fut le théâtre il y a de cela deux mille ans affectent aujourd'hui encore notre quotidien ». Et la notice finit sur cette interrogation inquiète : « Pourra-t-on un jour en dire autant des civilisations du XxI ${ }^{\mathrm{e}}$ siècle ?»

68. Civilopedia en ligne, s.v. "Cités-États ». La notice est introduite par une citation: " "L'homme apprend de la cité". Simonide. »

69. Civilization VI a ajouté des Cités-États religieuses, comme le Vatican, Jérusalem, Lhassa ou Katmandou. 
70. Il existe dix types de doctrines sociales, chacune ouvrant accès à un arbre de cinq politiques différentes. On peut déverrouiller l'une de ces politiques sociales, dès que l'on a acquis suffisamment de points de culture.

71. On remarque cependant que les doctrines sociales disponibles dès le début du jeu sont la Tradition, la Liberté (Liberty), l'Honneur et la Piété. Pour le Patronage et le Commerce, il faut attendre le Moyen Âge, tandis que le Rationalisme et la Liberté (Freedom) seront disponibles à la Renaissance, l'Ordre et l'Autocratie à partir de l'Ère Industrielle.

72. Aristote, Constitution d'Athènes. Le régime politique des Athéniens, trad. et éd. Michel Sève, Paris, 2006.

73. Il en existe en réalité bien d'autres. Même la rapidité du jeu (qui peut être configurée) est formulée en "termes antiques": la vitesse va de "quick» à " epic » ou "marathon » (du temps le plus rapide pour accomplir une action au temps le plus long).

74. On notera ici l'irruption discrète d'une référence polythéiste qui contribue à installer subrepticement l'univers vidéo-ludique dans un environnement «à l'antique ».

75. Traduction personnelle d'une explication en anglais donnée sur le site dédié au jeu Civilization $V$ : http://civilization.wikia.com/wiki/Golden_Age_(Civ5)

76. Le guide du jeu donne des exemples historiques d'Âge d'or: l'Italie pendant la Renaissance, les États-Unis pendant la seconde moitié du $\mathrm{xx}^{\mathrm{e}}$ siècle (Civ. V, p. 109). Il s'agit d'un moment où « la civilisation déborde d'énergie et de vitalité. Les hommes deviennent très productifs, les avancées technologiques très rapides, la culture enviée de tous " (traduction personnelle).

77. «Places that truly display the grand beauty of the natural world. In addition to providing wealth and production, Natural Wonders permanently increase happiness when first seen by a civilization » (Civ. V, guide in line, p. 35).

78. «Wonders are the spectacular buildings, inventions, and concepts that have stood the test of time and changed the world forever » (Civ. V, p. 110).

79. Un bon exemple des Merveilles Nationales est « l'épopée nationale ».

80. Pausanias, Périégèse, $\mathrm{V}, 10,1$ : «La Grèce est certainement pleine de merveilles, qui causent de l'admiration à ceux qui les voient ou qui en entendent parler ». Voir aussi Périégèse, V, 10, 2-10 et 11 (Temple d'Olympie) ; VII, 2, 6-9 (Temple d'Ephèse); VIII, 16 (Mausolée d'Halicarnasse) et Hérodote, Enquête II, 124-126 (sur la pyramide de Khéops) et I, 178-180 (sur les remparts de Babylone) ; Diodore de Sicile, Bibliothèque historique, II, 7-10 (jardins de Babylone) ; Strabon, Géographie, XIV, 2, 16 et XVI, 1, 5 (Mausolée d'Halicarnasse et jardins de Babylone).

81. La question du dedans et du dehors, de l'articulation entre le jeu et l'espace-temps du monde réel est fondamentale dans les travaux sur le jeu, depuis ceux de Huizinga (1988 [1938]). L'acceptation des règles conduit le joueur à investir l'espace et le temps pour leur attribuer une signification différente.

82. On pense ici à l'inventaire du patrimoine mondial de l'UNESCO, réactualisé chaque année depuis 1978, visant à recenser, sur tous les continents, un ensemble de biens culturels et naturels présentant un intérêt exceptionnel pour l'héritage commun de l'humanité. Mais d'autres listes existent, notamment celle des Sept Merveilles du monde moderne, établie par l'American Society of Civil Engineers et consacrant les monuments $\mathrm{du} \mathrm{xx}$ siècle. On y trouve le Tunnel sous la Manche, la Tour CN de Toronto, l'Empire State Building, le Golden Gate Bridge, le Barrage d'Itaipu, le Plan Delta et le Canal de Panama. 
83. Je prends ici le mot au sens de Roland Barthes (1957).

84. André et Lécole-Solnychkine 2011, p. 184 et 2013, p. 93.

85. Alpha Centauri est également le nom d'un autre jeu, qui se présente comme la suite de Civilization. Le scénario est basé sur la colonisation de la planète Chiron, entre 2100-2600, et sur la lutte entre des factions rivales, dont la « fédération spartiate » et « les filles de Gaïa ». On voit à quel point l'Antiquité est nécessaire à l'élaboration de ces fictions.

86. La construction du Projet Utopia est la dernière étape du jeu pour celui qui aspire à la victoire culturelle. Il faut pour cela avoir parcouru les cinq branches du panel de politiques sociales.

87. Le programme Apollo marque le début de la course à l'espace. Il permet la construction des pièces du vaisseau spatial. Ce terme fait évidemment référence au programme, lancé aux États-Unis dans les années 50, qui permit d'envoyer les premiers hommes sur la lune en 1969.

88. Sur les rapports entre Antiquité et science-fiction, voir Aziza 2011, p. 270-276. La question de la langue n'est cependant pas traitée.

89. Voir Civilization V, p.179. Cette capacité spécifique permet l'obtention d'un bonus à chaque destruction de campement barbare.

90. Lucain, Pharsale I, vers $255-256$ : « Les premiers, nous avons vu les Gaulois y pénétrer, les Cimbres s'y répandre, les Carthaginois fondre du haut des Alpes, les courses et la fureur des Teutons (furor teutonicus) désoler ces bords; toutes les fois que la Fortune insulte Rome, c'est ici le chemin de la guerre. »

91. 38 millions d'exemplaires de ce jeu ont été vendus depuis sa sortie.

92. Sur la fascination pour l'Histoire dans la création des jeux vidéo, voir Rabino 2013.

93. Voir par exemple Corbeil et D. Laveault 2013 ; Boutonnet et al. 2014 ; et, pour un bilan et une bibliographie récente, Joly-Lavoie et Yelle 2016, p. 19-24, qui prennent l'exemple de Civilization comme objet principal de leur recherche. Notons par ailleurs que Sid Meier's a récemment annoncé une version éducative de la série Civilization. Voir le blog: http://classcraft.com/blog/news/g4c-news/sid-meier-announces-civilization-edu/

94. C'est en s'appuyant précisément sur les stéréotypes véhiculés par le jeu, en particulier la représentation graphique des chefs barbares par opposition aux dirigeants de l'Empire romain tardif, que les enseignants ont cherché à développer chez leurs élèves des compétences liées aux méthodes de l'historien. L'analyse critique du scénario de Civilization V concernant la chute de l'Empire romain et l'expérience de déconstruction des stéréotypes menée avec les élèves sont décrites dans l'article de Joly-Lavoie et Yelle 2016 cité supra. Sur l'image du barbare confortée par les représentations populaires de l'histoire, voir aussi Dalongeville 2001 (réf. citée par F. Yelle). Plus récemment encore et dans un tout autre but, l'Université de Cambridge, au sein du Centre for the Study of Existential Risk (CSER), a développé un nouveau mode de jeu pour Civilization V: une apocalypse menée par des Intelligences Artificielles (IA) devenues incontrôlables. Ce mode, dénommé Superintelligence, est destiné à sensibiliser un large public aux dangers potentiels liés au développement de l'intelligence artificielle et à la complexité des décisions à prendre dans ce domaine. Voici le lien vers la page du CSER de l'Université de Cambridge consacré à ce nouveau « mod » du jeu Civilization V : https://www.cser.ac.uk/ news/civilization-v-video-game-mod-superintelligent-ai/ 


\section{RÉSUMÉS}

De nombreux jeux vidéo utilisent l'Antiquité dans leur scénario. L'un d'eux passionne " gamers» et internautes depuis vingt-cinq ans : «Civilization». Le principe ? Conduire une "civilisation» depuis «l'âge de pierre» jusqu'à l'époque contemporaine en choisissant parmi dix-huit «civilisations» proposées et en affrontant d'autres civilisations. Chaque étape de cette « évolution » permet d'acquérir de nouveaux « pouvoirs » et de « progresser » jusqu'à la victoire. Très sophistiqué du point de vue technologique, "Civilization » se présente comme une sorte de bric-à-brac culturel: affranchi de toute vraisemblance historique, il s'appuie sur un évolutionnisme et un ethnocentrisme décomplexés. Il affiche un impérialisme triomphant et porte à son comble l'idéologie du «choc des civilisations » puisqu'il s'agit, pour gagner, de combattre et d'anéantir l'autre. Or, ce jeu permet la construction et la transmission de stéréotypes, d'une part sur ce qui constitue "une civilisation» dans la pensée occidentale contemporaine, d'autre part sur la spécificité et l'apport supposé de chaque civilisation (ou peuple) à l'histoire universelle, enfin sur la place spécifique conférée à l'Antiquité gréco-romaine dans ce « grand récit » de l'Histoire de l'humanité.

À partir de ce jeu, qui par sa diffusion et son succès nous semble un important vecteur de représentations culturelles, nous entendons poser une série de questions: quels traits caractéristiques sont retenus et mis en avant pour faire de la Grèce et de Rome des « civilisations"? Quels avantages spécifiques ces deux « civilisations » sont-elles supposées avoir sur leurs concurrentes? On verra ainsi l'usage et le fonctionnement d'éléments topiques comme la " cité-État ", "l'Âge d'or » ou les « Merveilles ». On se demandera aussi comment les langues grecque et latine sont utilisées et détournées pour créer une langue spécifique (celle des jeux vidéo), un idiome partagé fabriquant un univers de fiction.

Many video games are using Antiquity in their scenario. One of them has been fascinating gamers and online players for almost 25 years: Civilization. Its principle? To choose one of 18 "civilizations" and lead it from "Stone Age» to contemporary era while confronting other civilizations. Each step of this « evolution » allows the player to acquire new possibilities and to progress until the final victory.

Very sophisticated in terms of technology, this video game is a sort of cultural odds and ends: free of any historical verisimilitude (Caesar can face Napoleon), Civilization draws on uninhibited evolutionism and ethnocentrism. It displays a triumphant imperialism and brings to a climax the theory (and ideology) of "the clash of civilizations", since winning supposes to fight and annihilate the other. Yet, this game allows the construction and transmission of stereotypes regarding not only the definition of civilization in the occidental contemporary thought, but also the characteristics and the supposed contribution of each civilization (or people) to the world's history and in particular the special role devoted to Greek and Roman Antiquity in that "great tale » of the « Human History ».

Considering that game as an historical object and as an important vector of cultural representations, we propose to ask a few questions: which specific elements are chosen and put forward to make the Ancient Rome and Greece two "civilizations"? What are the special advantages that help them outperform their competitors (the other civilizations)? In that respect, we analyze the use and functioning of topical items such as the «City States », « Golden 
Age » or the " Marvels ». This survey also explores the way Latin and Greek words are used and diverted in order to form a specific videogames' idiom, a shared language that creates a fictional universe.

INDEX

Keywords : videogame, antiquity, civilization, marvels, latin language, barbarian Mots-clés : jeu vidéo, antiquité, civilisation, merveille, latin, barbare

\section{AUTEUR}

EMMANUELLE VALETTE

Université Paris Diderot-Paris 7, ANHIMA UMR 8210 